\title{
Schwann cells expressing nociceptive channel TRPA1 orchestrate ethanol-evoked neuropathic pain in mice
}

\author{
Francesco De Logu, ${ }^{1}$ Simone Li Puma, ${ }^{1}$ Lorenzo Landini, ${ }^{1}$ Francesca Portelli, ${ }^{2}$ Alessandro Innocenti, ${ }^{3}$ \\ Daniel Souza Monteiro de Araujo, ${ }^{1,4}$ Malvin N. Janal, ${ }^{5}$ Riccardo Patacchini, ${ }^{6}$ Nigel W. Bunnett, ${ }^{7,8}$ Pierangelo Geppetti, ${ }^{1}$ \\ and Romina Nassini ${ }^{1}$ \\ ${ }^{1}$ Section of Clinical Pharmacology and Oncology, Department of Health Sciences, University of Florence, Florence, Italy. ${ }^{2}$ Histopathology and Molecular Diagnostics, Department of Health Sciences, \\ University of Florence, Florence, Italy. ${ }^{3}$ Plastic and Reconstructive Microsurgery, Careggi University Hospital, Florence, Italy. ${ }^{4}$ Department of Neurobiology and Program of Neurosciences, Institute of Biology, \\ Fluminense Federal University, Niterói, Brazil. 5Department of Epidemiology and Health Promotion, New York University College of Dentistry, New York, New York, USA. ${ }^{6}$ Department of Corporate Drug \\ Development, Chiesi Farmaceutici SpA, Parma, Italy. ${ }^{7}$ Department of Surgery and ${ }^{8}$ Department of Pharmacology, Columbia University in the City of New York, New York, New York, USA.
}

\begin{abstract}
Excessive alcohol consumption is associated with spontaneous burning pain, hyperalgesia, and allodynia. Although acetaldehyde has been implicated in the painful alcoholic neuropathy, the mechanism by which the ethanol metabolite causes pain symptoms is unknown. Acute ethanol ingestion caused delayed mechanical allodynia in mice. Inhibition of alcohol dehydrogenase (ADH) or deletion of transient receptor potential ankyrin 1 (TRPA1), a sensor for oxidative and carbonyl stress, prevented allodynia. Acetaldehyde generated by ADH in both liver and Schwann cells surrounding nociceptors was required for TRPA1-induced mechanical allodynia. PIp1-Cre Trpa1 ${ }^{f l / f l}$ mice with a tamoxifen-inducible specific deletion of TRPA1 in Schwann cells revealed that channel activation by acetaldehyde in these cells initiates a NADPH oxidase-1-dependent (NOX1dependent) production of hydrogen peroxide $\left(\mathrm{H}_{2} \mathrm{O}_{2}\right)$ and 4-hydroxynonenal (4-HNE), which sustains allodynia by paracrine targeting of nociceptor TRPA1. Chronic ethanol ingestion caused prolonged mechanical allodynia and loss of intraepidermal small nerve fibers in WT mice. While Trpa1 ${ }^{-/-}$or PIp1-Cre Trpa fl/fl mice did not develop mechanical allodynia, they did not show any protection from the small-fiber neuropathy. Human Schwann cells express ADH/TRPA1/NOX1 and recapitulate the proalgesic functions of mouse Schwann cells. TRPA1 antagonists might attenuate some symptoms of alcohol-related pain.
\end{abstract}

\section{Introduction}

Alcohol abuse and dependence are among the major health care problems in the world (1). The impact of alcohol abuse continues to grow as a result of the rising incidence of excessive drinking by women and young people (2). Nevertheless, the male/female ratio of current drinkers and heavy episodic drinkers, which is 1.7 and 2.5, respectively, indicates twice the prevalence in men compared with women (2). About $60 \%$ of alcoholics exhibit a peripheral polyneuropathy, associated with pain and dysesthesias of the lower extremities (3). Reduced thiamine levels (4), which cause peripheral neuropathy in patients with postgastrectomy polyneuropathy or beriberi, have been reported in alcoholics, and have been considered responsible for the peripheral neuropathy $(5,6)$. However, the observation that painful polyneuropathy is present in alcoholics with normal thiamine status (6), and that the clinical and pathological features of alcoholic neuropathy are distinct from those associated with thiamine deficiency (7), led to the conclusion that alcohol exerts a direct toxic effect on peripheral nerves. However, the mechanism by which ethanol targets peripheral nerves to cause the painful condition remains unknown.

Conflict of interest: RP is fully employed at Chiesi Farmaceutici SpA, Parma, Italy. NWB is a founding scientist of Endosome Therapeutics Inc. Copyright: (5) 2019, American Society for Clinical Investigation. Submitted: February 11, 2019; Accepted: August 29, 2019; Published: November 4, 2019 Reference information: / Clin Invest. 2019;129(12):5424-5441.

https://doi.org/10.1172/JCI128022.
Alcohol dehydrogenase (ADH) converts ethanol into the reactive and toxic product acetaldehyde, which is rapidly metabolized to acetic acid by the mitochondrial aldehyde dehydrogenase-2 (ALDH2) (8). Acetaldehyde is considered as the major contributor of the detrimental effects produced by acute and chronic alcohol consumption, including flushing, headache, cirrhosis, and cancer (9-12). Mutations of the ADH and ALDH2 genes, which respectively accelerate ethanol conversion to acetaldehyde and slow down acetaldehyde metabolism, make East Asians susceptible to alcohol-induced symptoms with lower rates of alcohol use (13, 14). Acetaldehyde generates advanced glycation end products and increases oxidative stress, which can contribute to ethanolinduced painful neuropathy (15). In particular, preclinical studies and clinical evidence have implicated acetaldehyde in the genesis of the neuronal damage associated with ethanol consumption (3). However, the pathways by which acetaldehyde causes ethanolevoked neuropathic pain are poorly understood.

Elevated local concentrations (1\%-3\%) of ethanol have been shown to activate the transient receptor potential vanilloid 1 (TRPV1) channel on primary sensory nerves to induce pain and neurogenic inflammation (16). A related channel, TRP ankyrin 1 (TRPA1), is uniquely sensitive to multiple reactive oxygen, nitrogen, and carbonyl species (ROS, RNS, and RCS, respectively) (17-19). Saturated and unsaturated aldehydes, including acetaldehyde and 4-hydroxynonenal (4-HNE), also activate TRPA1 to cause pain-like responses $(20,21)$. TRPV1, TRPA1, and other TRP channels, including the vanilloid 4 (TRPV4), are expressed 
by a subpopulation of primary sensory neurons that signal pain (19). In the present study, we explored the role of TRP channels in the pain-like responses evoked by acute and chronic ethanol ingestion in mice.

We found that local (intraplantar) injection of ethanol in the mouse hind paw caused an acute nociceptive response due to direct activation of TRPV1, as previously reported (16). In addition, ethanol (either intraplantar or intragastric) caused a delayed and prolonged TRPV1-independent mechanical allodynia of the hind paw that was prevented by ADH and TRPA1 inhibition. Surprisingly, Schwann cells that ensheath plantar nerve fibers expressed $\mathrm{ADH}$, which mediated the local conversion of ethanol to acetaldehyde. Selective deletion of TRPA1 on Schwann cells or nociceptors revealed that acetaldehyde, generated in the liver or locally, activates TRPA1 on Schwann cells (22) to produce oxidative and carbonylic stress, which targets neuronal TRPA1 to sustain allodynia. Notably, mice fed daily with an ethanol-containing diet, which mimics chronic alcohol ingestion by alcoholics, developed a prolonged allodynia that was associated with loss of intraepidermal nerve fibers. Allodynia caused by chronic ethanol ingestion was prevented by the same genetic and pharmacological disruptions of TRPA1 and oxidative stress that attenuated allodynia evoked by acute ethanol exposure. Thus, TRPA1 antagonism might represent an effective treatment for pain in alcoholics.

\section{Results}

TRPV1 mediates acute nociception but not sustained allodynia in response to local administration of ethanol. To explore the mechanisms of alcohol-induced nociception, ethanol was administered locally to the hind paw of C57BL/6J mice. Injection of intraplantar ethanol $(15 \%-80 \%$ in $0.9 \% \mathrm{NaCl})$ caused dose-dependent immediate nociceptive behavior, which did not last more than 5 minutes (Figure 1A). Ethanol also produced a delayed and prolonged (1-6 hours) mechanical allodynia (Figure 1B). The short-lived nociceptive response was absent in $\operatorname{Trpv1}^{-/-}$mice (Figure 1C), and in C57BL/6J mice pretreated with the TRPV1 antagonist SB366791 (Supplemental Figure 1A; supplemental material available online with this article; https://doi.org/10.1172/JCI128022DS1), supporting previous evidence that ethanol selectively targets TRPV1 to signal acute pain (16). In contrast, TRPV1 deletion or antagonism did not affect ethanol-evoked mechanical allodynia (Figure 1D and Supplemental Figure 1B). Thus, whereas direct TRPV1 engagement mediates acute ethanol-evoked nociception, ethanol induces sustained mechanical allodynia by a TRPV1-independent mechanism. Ethanol-induced nociception and allodynia were similar in Trpv $4^{-/-}$ mice and in WT littermates (Supplemental Figure 1, C and D).

Acetaldehyde and TRPA1 mediate sustained allodynia in response to local and intragastric administration of ethanol. ADH mediates the first step of alcohol metabolism, converting ethanol to the saturated aldehyde acetaldehyde, which can activate TRPA1 (20). We examined the contribution of ADH and TRPA1 to ethanol-evoked allodynia. Intragastric or intraplantar pretreatment of C57BL/6J mice with 4-methylpyrazole (4-Mp), a selective $\mathrm{ADH}$ inhibitor, prevented ethanol-evoked allodynia (Figure 1, E and F). The nociceptive response evoked by intraplantar ethanol observed in Trpa1 $1^{+/+}$mice was also present in Trpa1 $1^{-/-}$mice (Figure 1G). However, mechanical allodynia produced by intraplantar ethanol in
$\operatorname{Trpa1}^{+/+}$mice was absent in Trpa1/- mice (Figure $1 \mathrm{H}$ ). Systemic (i.p.) or intraplantar administration of A967079, a TRPA1 antagonist, 2 hours after ethanol reversed sustained allodynia (Supplemental Figure 1, E and F). Intraplantar injection of acetaldehyde (1-20 nmol) into the hind paw of C57BL/6J mice replicated the actions of ethanol, causing a dose-dependent immediate and transient nociceptive response (Figure 1I), followed by a delayed and prolonged (1-8 hours) allodynia (Figure 1J). Both responses were absent in Trpa1 1 - mice (Figure 1, K and L). Systemic (i.p.) or intraplantar administration of A967079 reversed immediate nociception and sustained allodynia evoked by intraplantar acetaldehyde in C57BL/6J mice (Supplemental Figure 1, G-I). Furthermore, the acute nociceptive response and the delayed mechanical allodynia induced by intraplantar acetaldehyde in Trpv1 ${ }^{+/+}$mice remained unaffected in Trpv1/- mice (Supplemental Figure 1, J and $\mathrm{K}$ ). The results are consistent with the hypothesis that ethanol induces allodynia by a mechanism that entails metabolism to acetaldehyde, which activates TRPA1.

Examination of the responses evoked by local injection of ethanol or acetaldehyde provides mechanistic insights into alcohol-induced pain. However, the ingestion of alcohol causes peripheral neuropathy in alcoholics. To mimic these circumstances, we administered graded doses of ethanol $(1-4 \mathrm{~mL} / \mathrm{kg}$ of ethanol $15 \%$ in $0.9 \% \mathrm{NaCl}$ ) to mice intragastrically. Even at the highest dose $(15 \%, 4 \mathrm{~mL} / \mathrm{kg}$, intragastric), ethanol did not induce acute nociception or affect motor coordination, evaluated using a rotarod: no falls were observed in mice receiving either vehicle $(n=8)$ or ethanol $(15 \%, 4 \mathrm{~mL} / \mathrm{kg}, n=8)$. However, intragastric ethanol evoked a dose-related delayed and sustained (1-6 hours) mechanical allodynia in C57BL/6J mice and Trpa1 $1^{+/+}$mice, but not in Trpa1 $^{-/-}$mice (Figure 2, A and B). Ethanol-evoked mechanical allodynia was preserved in $\operatorname{Tr} p v 1^{-/-}$and $\operatorname{Tr} p v 4^{-/-}$mice (Supplemental Figure 1, L and M). Systemic (i.p.) or intraplantar administration of A967079 to C57BL/6J mice reversed allodynia evoked by intragastric ethanol (Figure 2, C and D).

After gavage, ethanol levels in plasma, liver, and paw were maximal at 30 minutes, and declined to baseline at 3 hours (Figure 2E), when mechanical allodynia was maximal (e.g., Figure 2A). The temporal dissociation between ethanol levels in plasma and tissues and allodynia suggests that ethanol does not directly activate TRPA1 to cause allodynia. Administration of the ADH inhibitor 4-Mp (intragastric) before ethanol prevented allodynia (Figure 2F). These results suggest that acetaldehyde, but not ethanol, activates TRPA1 to initiate allodynia.

Schwann cell ADH converts ethanol to acetaldehyde in peripheral tissues. Ethanol is mainly converted to acetaldehyde by $\mathrm{ADH}$ in the liver. However, ADH is expressed in other tissues (23-26). We detected mRNAs encoding ADH1, ADH5, and ADH7 in homogenates of mouse paw (Figure 3A). Immunoreactivity of $\mathrm{ADH}$ was confined to the nerve trunk within the paw, where it was coexpressed with S100, a specific marker of Schwann cells, and was not seen in PGP9.5-positive nerve fibers (Figure 3, B and C, and Supplemental Figure 2A). Exposure of cultured mouse Schwann cells to ethanol induced the generation of acetaldehyde, a response that was prevented by 4-Mp (Figure 3D). Local (intraplantar) administration of ethanol to the paw also resulted in increased paw tissue levels of acetaldehyde within 15 minutes 
A

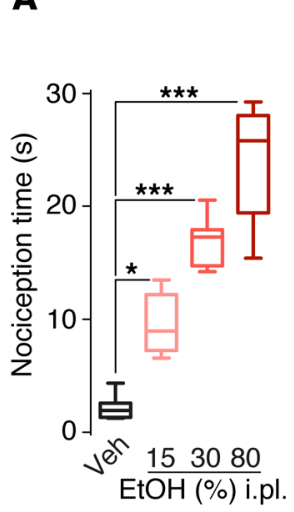

E

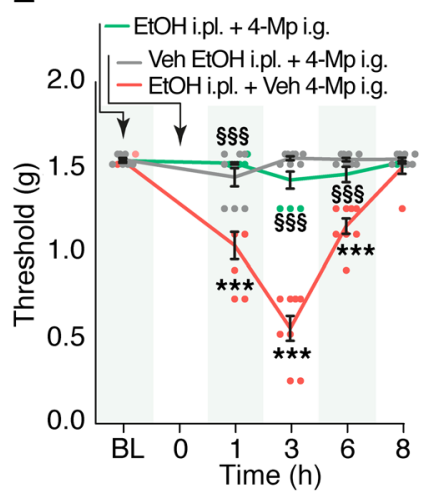

B

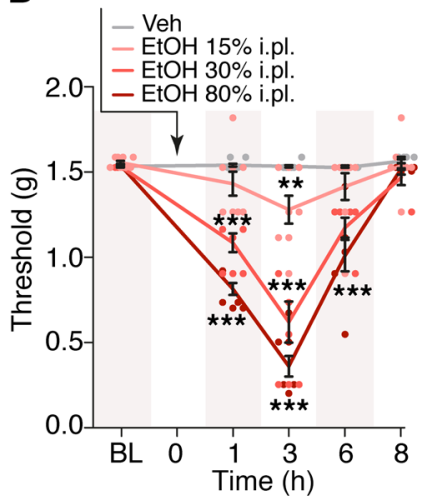

C

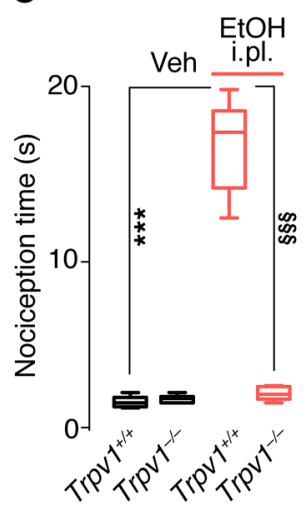

$\mathbf{F}$

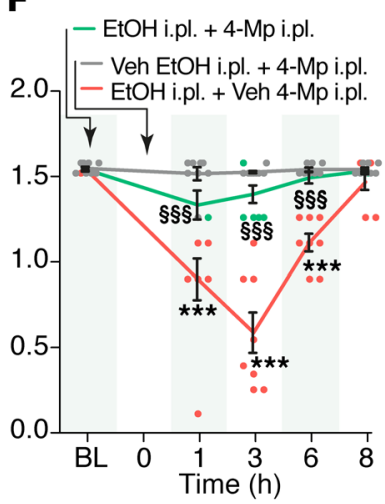

D

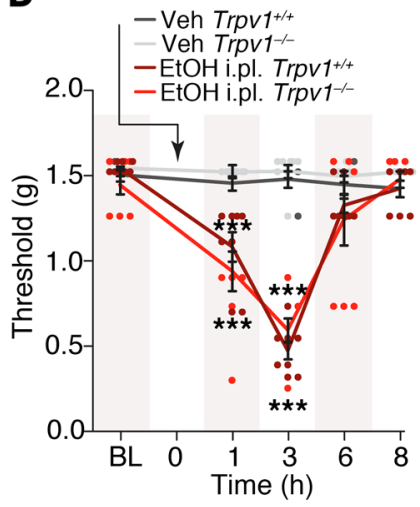

G

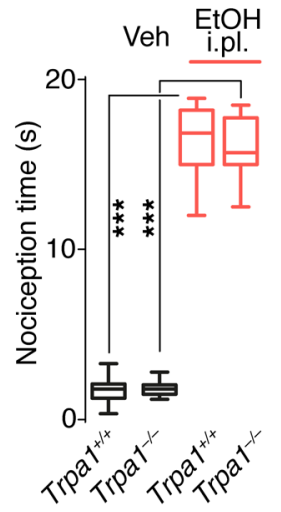

H $\quad$ - Veh Trpa1+t+

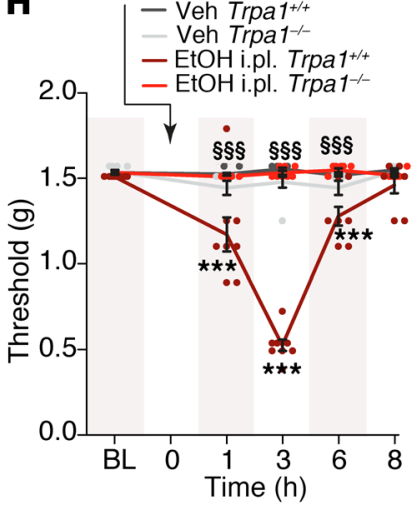

I

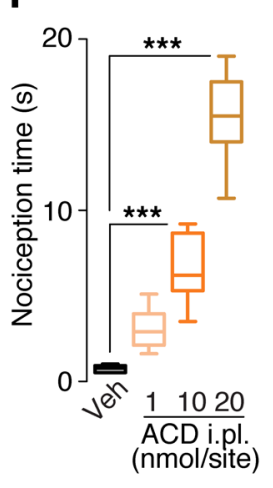

J

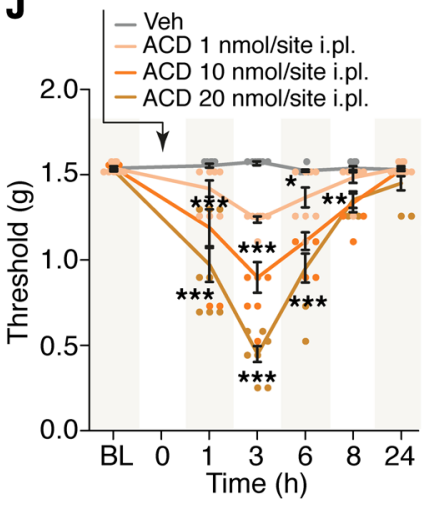

$\mathbf{K}$

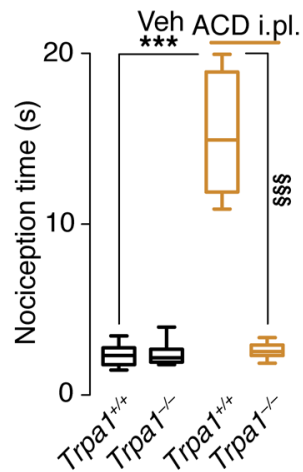

$\mathbf{L}$

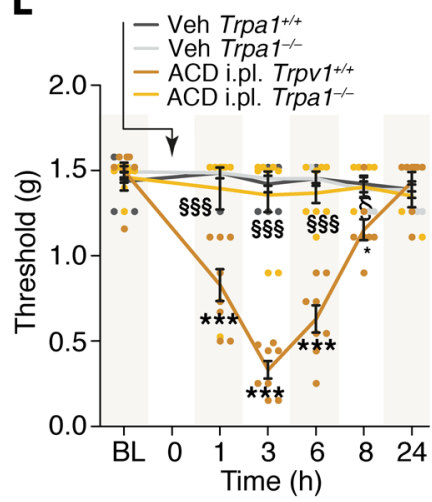

Figure 1. TRPV1 mediates acute nociception and TRPA1 mediates allodynia evoked by local ethanol. (A and B) Dose-dependent acute nociception (A) and dose- and time-dependent mechanical allodynia (B) evoked by intraplantar (i.pl., $20 \mu \mathrm{L}$ ) ethanol (EtOH) or vehicle (Veh) in C57BL/6) mice. (C) Acute nociceptive response evoked by EtOH (30\%, i.pl.) or Veh in Trpv1 $7^{+/+}$and $\operatorname{Trpv1}^{-/-}$mice. (D) Time-dependent mechanical allodynia evoked by EtOH (30\%, i.pl.) or Veh in Trpv1+/+ and Trpv1//- mice. (E and F) Time-dependent mechanical allodynia evoked by EtOH (30\%, i.pl.) or Veh in C57BL/6) mice pretreated with 4-methylpyrazole (4-Mp; $50 \mathrm{mg} / \mathrm{kg}$, intragastric [i.g.], or $100 \mu$ g, i.pl.) or Veh 4-Mp. (G and $\mathbf{H})$ Acute nociceptive response (G) and time-dependent mechanical allodynia (H) evoked by EtOH (30\%, i.pl.) or Veh in Trpa1//+ and Trpa1/- mice. (I and J) Dose-dependent acute nociceptive response (I) and dose- and time-dependent mechanical allodynia (J) evoked by acetaldehyde (ACD; 1-20 nmol, i.pl.) or Veh in C57BL/6) mice. (K and $\mathbf{L}$ ) Acute nociceptive response and

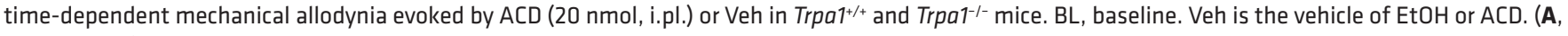
C, G, I, and $\mathbf{K}$ ) Box plots with horizontal lines at the 25th percentile, the median, and the 75th percentile and vertical lines that extend to the minimum and maximum values; (B, D-F, H, J, and $\mathbf{L}$ ) mean $\pm \mathrm{SEM}$ with individual data points overlaid; $n=8$ mice for each experiment. ${ }^{*} P<0.05$, ${ }^{* *} P<0.01,{ }^{* * *} P<0.001$

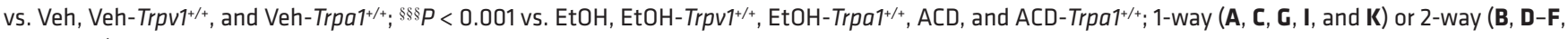
$\mathbf{H}, \mathbf{J}$, and L) ANOVA with Bonferroni post hoc correction.

(Figure 3E). The local (intraplantar) administration of 4-Mp inhibited acetaldehyde formation (Figure 3F) and allodynia (Figure $1 F$ ) induced by intraplantar ethanol. The effects of intragastric ethanol on allodynia and acetaldehyde levels were partially reduced by pretreatment with intraplantar (Figure 3, G and H), or completely attenuated by intragastric, 4-Mp (Figure $3 \mathrm{H}$ and Figure $2 \mathrm{~F})$. These results suggest that Schwann cells in the paw express $\mathrm{ADH}$ and convert ethanol to acetaldehyde. This local generation 
A

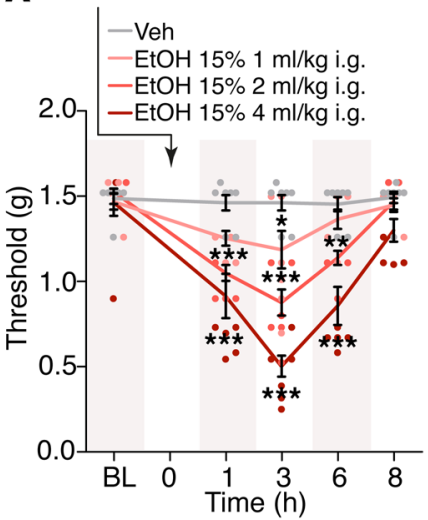

C
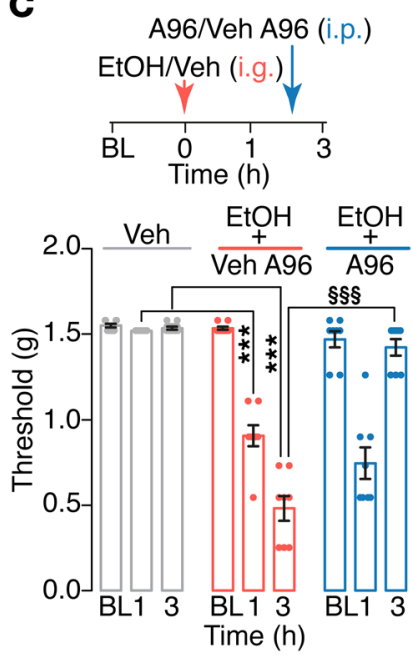

E

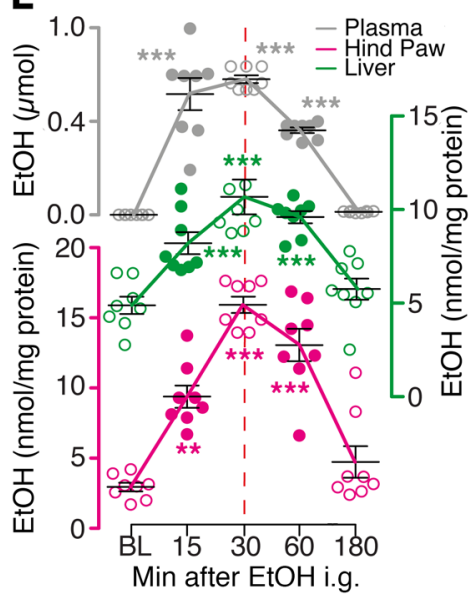

B

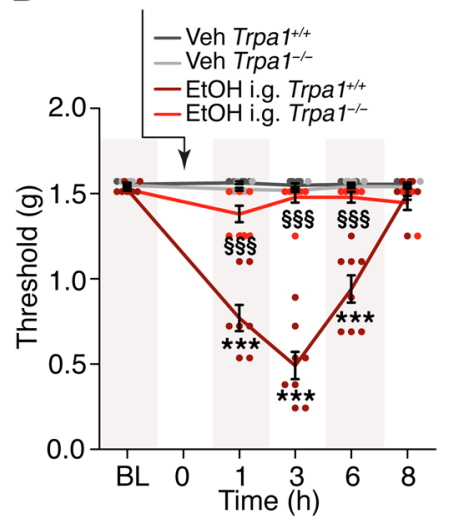

D
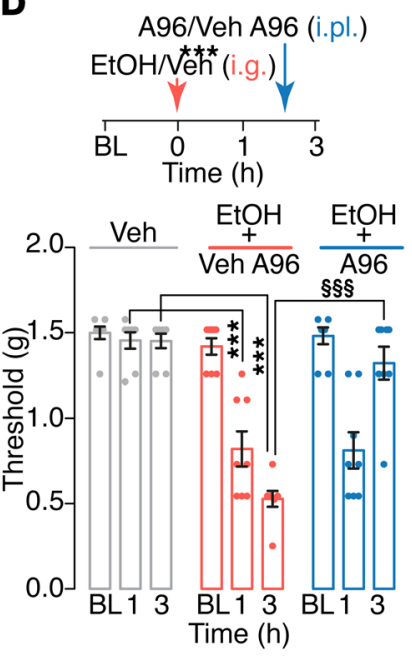

$\mathbf{F}$

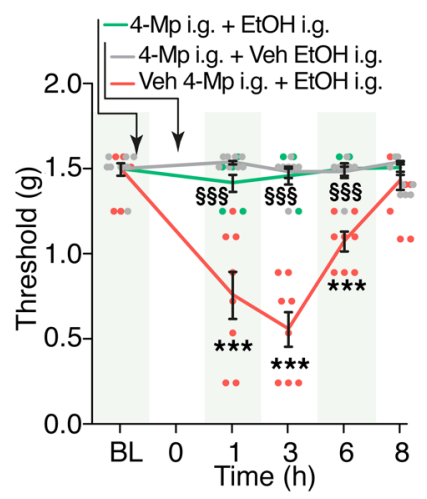

Figure 2. TRPA1 mediates mechanical allodynia evoked by intragastric ethanol in C57BL/6J mouse hind paw. (A) Dose-dependent mechanical allodynia evoked by ethanol (EtOH, i.g.) or vehicle (Veh). (B) Time-dependent mechanical allodynia evoked by EtOH $(15 \%, 4 \mathrm{~mL} / \mathrm{kg}$, i.g.) or Veh in $\mathrm{Trpa}^{1^{+/}}$and $\mathrm{Trpa1}^{-/-}$mice. (C and D) Mechanical allodynia evoked by EtOH (15\%, $4 \mathrm{~mL} / \mathrm{kg}$, i.g.) or Veh after A967079 (A96, $100 \mathrm{mg} / \mathrm{kg}$, intraperitoneal [i.p.], or $100 \mu \mathrm{g} / \mathrm{site}$, i.pl.) or Veh $\mathrm{A} 96$ in [57BL/6) mice. (E) Time course of EtOH levels in plasma and liver and hind-paw tissue after EtOH (15\%, $4 \mathrm{~mL} / \mathrm{kg}$, i.g.) in [57BL/6) mice. (F) Time-dependent mechanical allodynia evoked by EtOH (15\%, $4 \mathrm{~mL} / \mathrm{kg}$, i.g.) or Veh in C57BL/6) mice pretreated with 4-Mp (50 mg/kg, i.g.) or Veh 4-Mp. BL, baseline. Veh is the vehicle of EtOH. Data are mean \pm SEM with individual data points overlaid; $n=8$ mice for each experimental condition. ${ }^{*} P<0.05$, ${ }^{* *} P<$ $0.01,{ }^{* * *} P<0.001$ vs. Veh and EtOH BL; ${ }^{\$ \S} P<0.001$ vs. EtOH-Trpa1 ${ }^{+/+}$, EtOH-A96, and 4-Mp-EtOH; 1-way (E) or 2-way (A-D and F) ANOVA with Bonferroni post hoc correction.

evoked a dose-dependent, delayed, and prolonged (1-8 hours) mechanical allodynia in C57BL/6J mice (Figure 4A). Acetaldehyde did not cause allodynia in Trpa1 $1 /$ mice (Figure 4B). Systemic (i.p.) and intraplantar A967079 prevented acetaldehyde-evoked allodynia in C57BL/6J mice (Figure 4, C and D). However, acetaldehyde formation in liver and paw after intragastric ethanol did not coincide with allodynia (Figure 4E); the levels of acetaldehyde in the liver and paw had returned to baseline by 3 hours after intragastric ethanol (Figure 4E), when allodynia was robustly maintained (Figure 2A). Furthermore, administration of the ADH inhibitor 4-Mp (intragastric) after ethanol did not affect allodynia (Figure 4F). These results suggest the existence of additional mechanisms of ethanolinduced allodynia.

Both ethanol and acetaldehyde can release ROS (27-29). ROS and their carbonylic by-products, including 4-HNE, activate TRPA1 $(17,21)$. To examine the contribution of ROS to ethanol-evoked allodynia, we treated mice with the ROS scavenger phenyl- $\alpha$-tert-butyl nitrone (PBN). PBN (i.p. or intraplantar) reversed the allodynia evoked by ethanol (both intraplantar and intragastric) (Figure 4, G and H, and Supplemental Figure 3, A and B) or acetaldehyde (Figure 4, I and J, and Supplemental Figure 3, C and D). Ethanol (intragastric or intraplantar) increased hydrogen peroxide $\left(\mathrm{H}_{2} \mathrm{O}_{2}\right)$ and 4-HNE levels in the paw (Figure 5, A and B). However, while $\mathrm{H}_{2} \mathrm{O}_{2}$ levels were elevated for 3 hours, 4-HNE content, measured by either immunofluorescence or a biochemical assay, persisted for 6 hours (Figure 5, A and B). To test the hypothesis that the late phase of allodynia was mainly due to RCS, $N$-acetylcysteine (NAC), which efficiently scavenges $\alpha, \beta$ unsaturated aldehydes, including 4-HNE (30), was given (i.p.) to mice 5 hours after ethanol (intragastric). NAC (i.p.) attenuated mechanical allodynia at 6 hours (Figure 5C). These results suggest that carbonylic derivatives, which are more stable and longer-acting than ROS (30), sustain the final phase of allodynia. To support this hypothesis, PBN, which selectively scavenges ROS, was ineffective in the final phase of allodynia (Figure 5C).

Administration of the ADH inhibitor 4-Mp (Figure 5D) or the ROS scavenger PBN (Figure 5E) blunted the ethanolinduced increase in $\mathrm{H}_{2} \mathrm{O}_{2}$ levels. Acetaldehyde (i.p. or intraplantar) (i.p.) did not cause detectable acute nociceptive behavior, but 

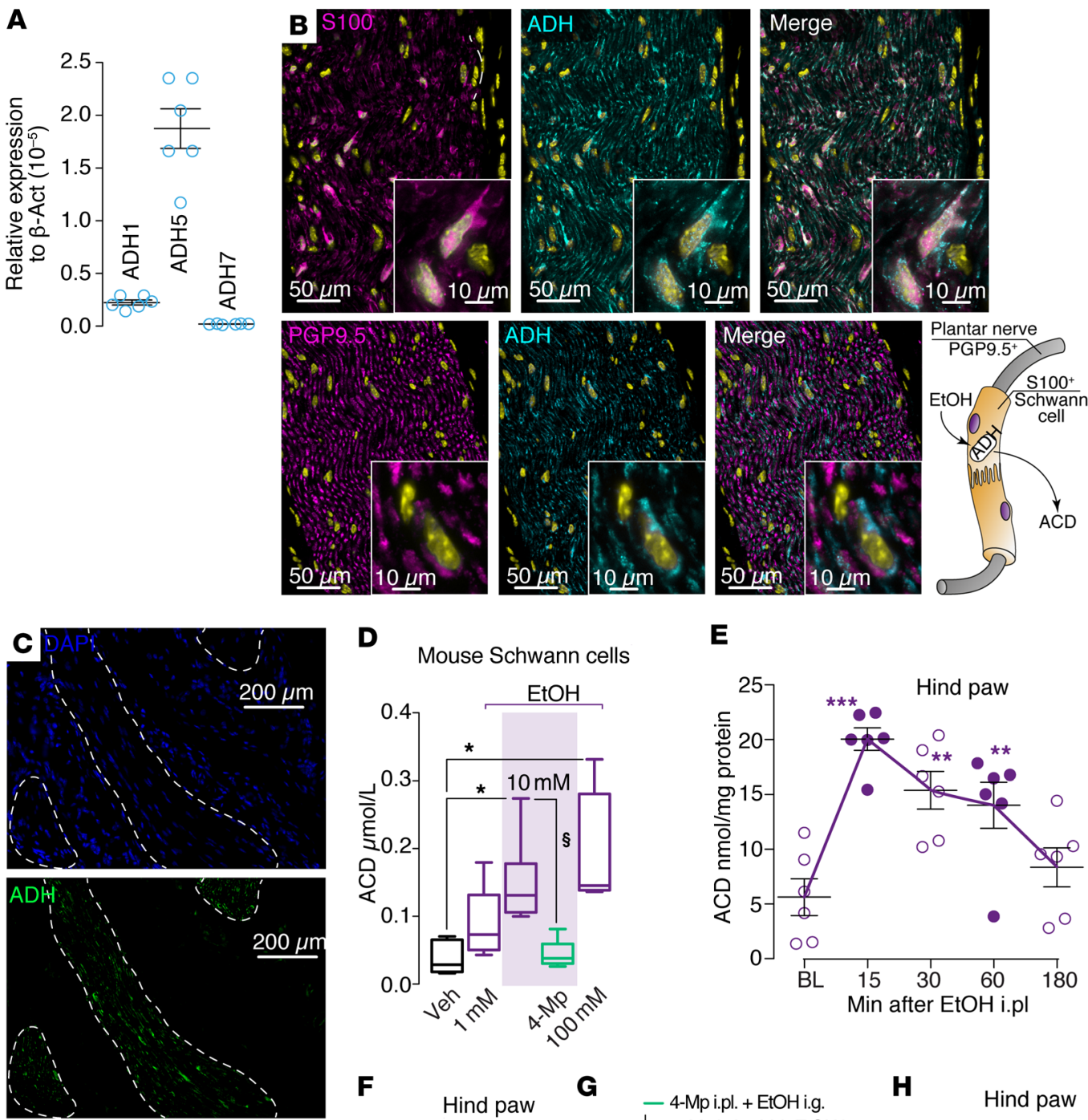

$\mathbf{E}$
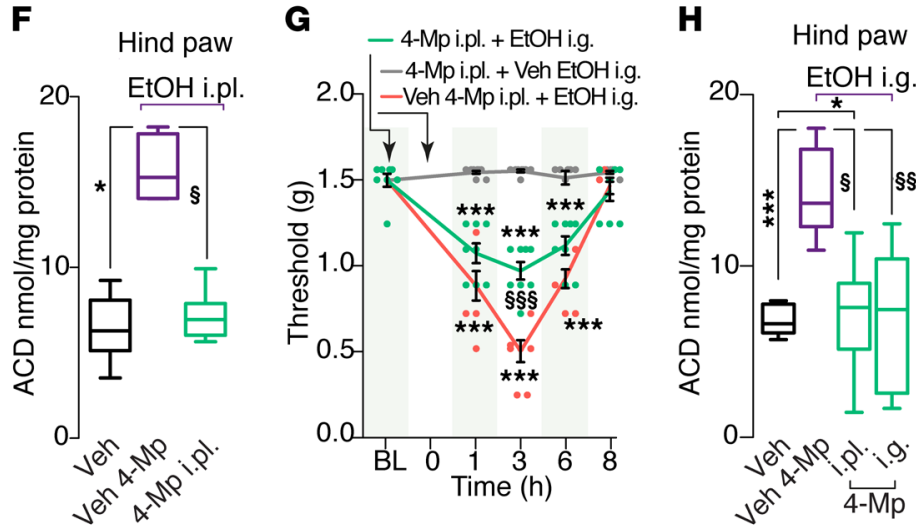

Figure 3. Acetaldehyde is generated by both hepatic and Schwann cell ADH. (A) ADH1, ADH5, and ADH7 mRNA relative expression in hind-paw tissue in C57BL/6) mice. (B and C) Representative images of ADH, S100, and PGP9.5 expression in hind-paw tissue in C57BL/6) mice. (D) Dose-dependent acetaldehyde (ACD) levels in cultured Schwann cells isolated from [57BL/6) mice and exposed to ethanol (EtOH; 1-100 mM) in the presence of 4-Mp $(100 \mu \mathrm{M})$ or vehicle (Veh 4-Mp). (E) Time-dependent ACD levels (hind-paw tissue) after i.pl. (20 $\mu \mathrm{L}) \mathrm{EtOH}(30 \%)$ in C57BL/6) mice. (F) ACD levels (hind-paw tissue) of [57BL/6) mice receiving EtOH (30\%, i.pl.) or Veh and pretreated with 4-Mp (100 $\mu$ g, i.pl.) or Veh 4-Mp. (G and $\mathbf{H})$ Time-dependent mechanical allodynia and ACD levels (hind-paw tissue) in C57BL/6) mice receiving EtOH (15\%, $4 \mathrm{~mL} / \mathrm{kg}$, i.g.) or Veh and pretreated with 4-Mp (100 $\mu$ g, i.pl., or $50 \mathrm{mg} / \mathrm{kg}$, i.g.) or Veh 4-Mp. BL, baseline. Veh is the vehicle of EtOH. "In" and "Out" indicate inside and outside, respectively, the perineurium, delimited by dashed lines. ( $\mathbf{D}, \mathbf{F}$, and $\mathbf{H}$ ) Box plots with horizontal lines at the 25 th percentile, the median, and the 75th percentile and vertical lines that extend to the minimum and maximum values; (A, E, and G) mean \pm SEM with individual data points overlaid; $n=6-8$ mice for each experimental condition. ${ }^{*} P<0.05,{ }^{* *} P<0.01,{ }^{* * *} P<0.001$ vs. Veh and ACD BL; ${ }^{\circledR} P<0.05,{ }^{\S \S} P<0.01,{ }^{\S \S} P<0.001$ vs. EtOH and EtOH-4-Mp; $1-w a y(\mathbf{D}-\mathbf{F}$ and $\mathbf{H})$ or 2-way (G) ANOVA with Bonferroni post hoc correction. 
A

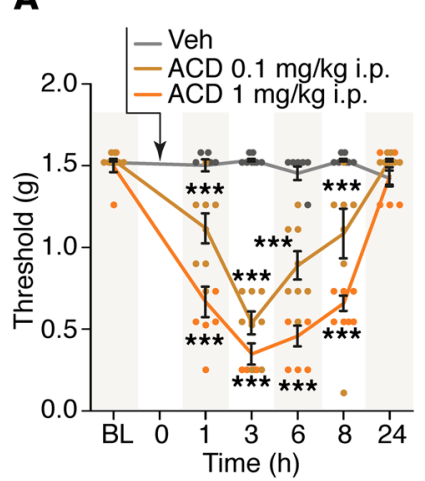

B

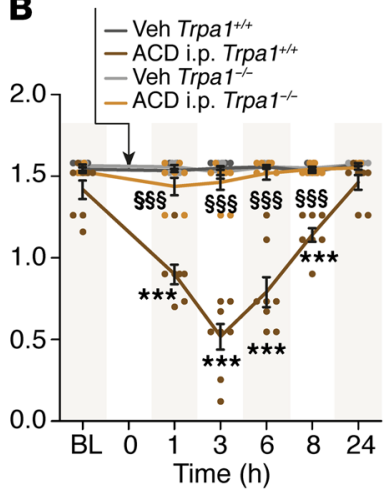

D

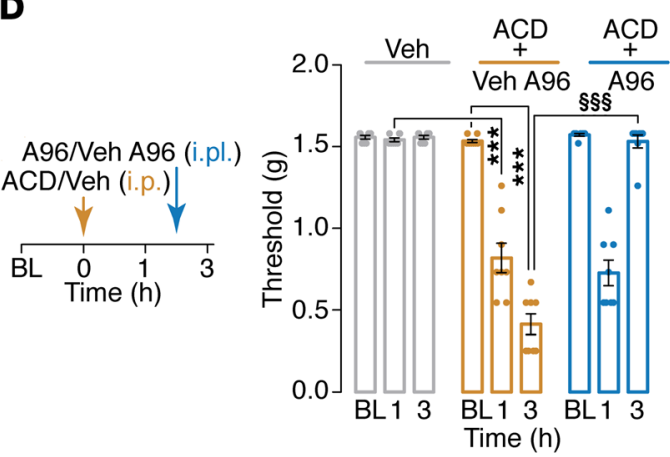

E

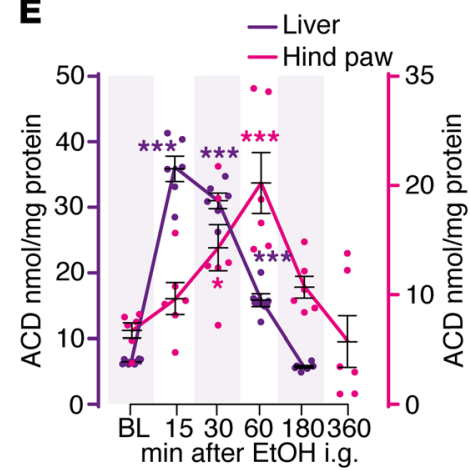

I
C
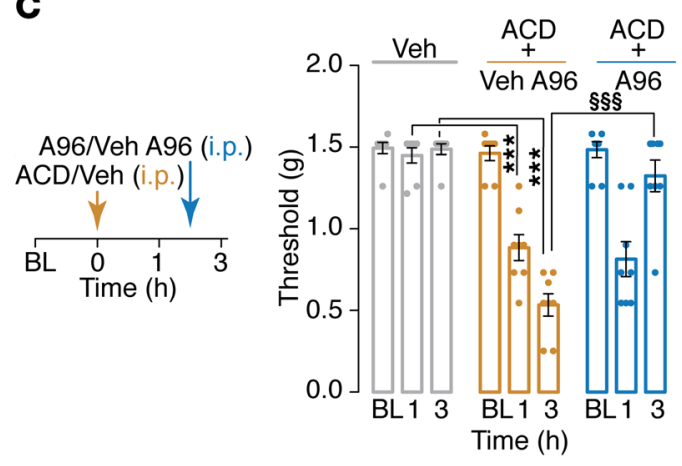

$\mathbf{F}$

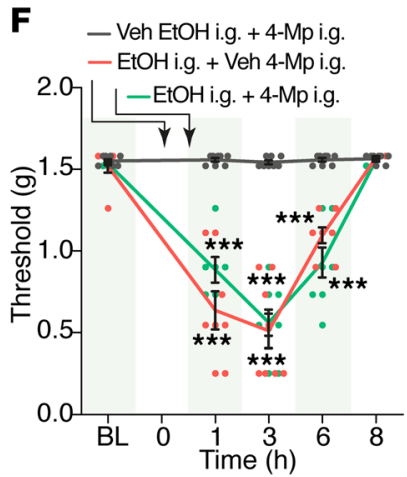

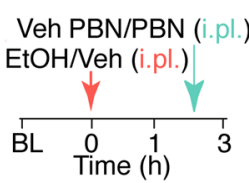

$\mathrm{EtOH} \mathrm{EtOH}$

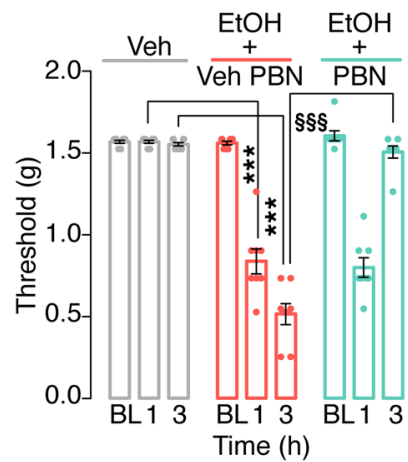

H
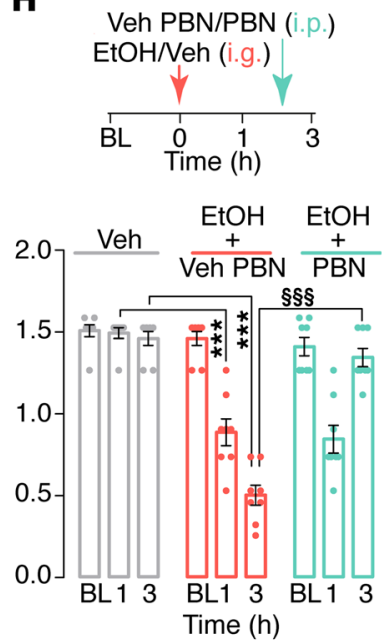

G

Figure 4. Acetaldehyde via TRPA1 sustains ethanol-evoked allodynia in C57BL/6J mouse hind paw. (A) Dose- and time-dependent mechanical allodynia

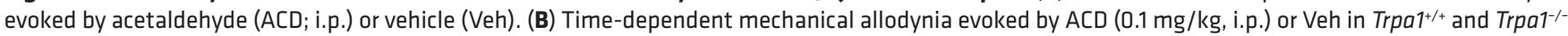
mice. (C and D) Mechanical allodynia evoked by ACD (0.1 mg/kg, i.p.) or Veh after A967079 (A96, $100 \mathrm{mg} / \mathrm{kg}$, i.p., or $100 \mu$ g, i.pl.) or Veh A96 in C57BL/6) mice. (E) Time-dependent ACD levels in liver and hind-paw tissue after ethanol (EtOH; $15 \%, 4 \mathrm{~mL} / \mathrm{kg}$, i.g.) in C57BL/6) mice. (F) Effect of post-treatment with 4-Mp (50 mg/kg, i.g.) or Veh 4-Mp on mechanical allodynia evoked by EtOH (15\%, $4 \mathrm{~mL} / \mathrm{kg}$, i.g.) or Veh in C57BL/6) mice. (G and H) Mechanical allodynia evoked by EtOH (30\%, i.pl., and $15 \%, 4 \mathrm{~mL} / \mathrm{kg}$, i.g.) Veh after phenyl- $\alpha$-tert-butyl nitrone (PBN, $100 \mu$ g, i.pl., or $50 \mathrm{mg} / \mathrm{kg}$, i.p.) or Veh PBN in C57BL/6] mice. (I and J) Mechanical allodynia evoked by ACD (10 nmol, i.pl., and $0.1 \mathrm{mg} / \mathrm{kg}$, i.p.) or Veh after PBN (100 $\mu$ g, i.pl., or $50 \mathrm{mg} / \mathrm{kg}$, i.p.) or Veh PBN in [57BL/6] mice. BL, baseline. Veh is the vehicle of EtOH and ACD. Data are mean \pm SEM with individual data points overlaid; $n=6-8$ mice for each experimental condition. ${ }^{*} P<0.05,{ }^{* * *} P<0.001$ vs. Veh and ACD BL; ${ }^{\$ \S} P<0.001$ vs. ACD-Trpa1 ${ }^{+/+}, A C D-A 96$, EtOH-PBN, and ACD-PBN; $1-$ way (E) or 2-way (A-D and F-J) ANOVA with Bonferroni post hoc correction. increased $\mathrm{H}_{2} \mathrm{O}_{2}$ in paw tissue (Figure 5F), an effect that was inhibited by PBN (Figure 5G). Surprisingly, we observed that pharmacological blockade or genetic deletion of TRPA1 attenuated both ethanol- and acetaldehyde-evoked increases in $\mathrm{H}_{2} \mathrm{O}_{2}$ in the paw (Figure 5, E and G-I). We have recently reported the presence of different NADPH oxidase (NOX) isoforms in Schwann cells of the mouse sciatic nerve, where they colocalize with TRPA1 to increase oxidative stress (22). Here, we observed that Schwann cells of plantar nerve fibers expressed NOX1 and TRPA1 (Figure 6, A and B, and Supplemental Figure 2A). A NOX1 antagonist (ML171, i.p.) 
$\mathbf{A}$

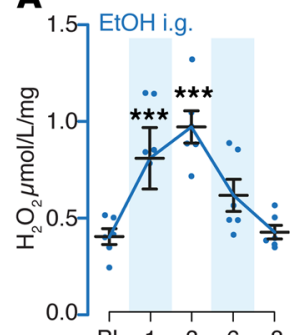

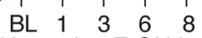
Hour after EtOH i.g.

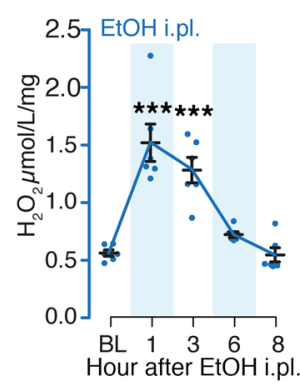

D
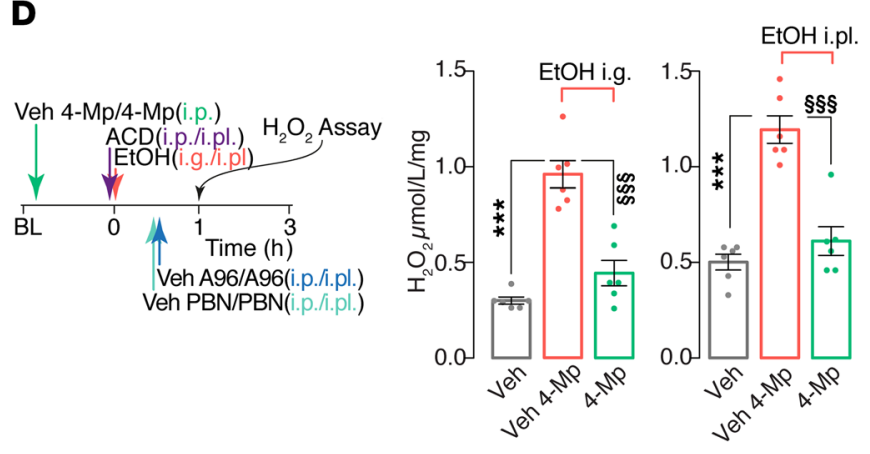

B
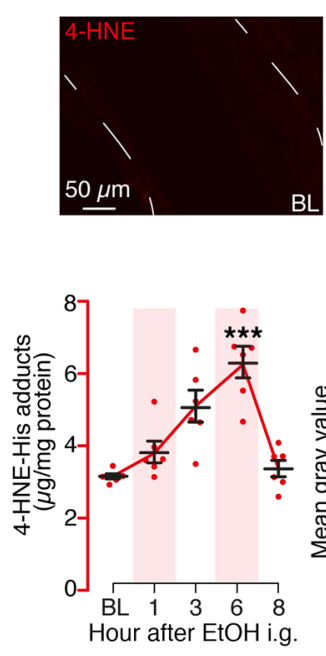

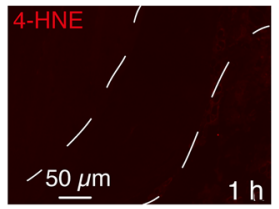

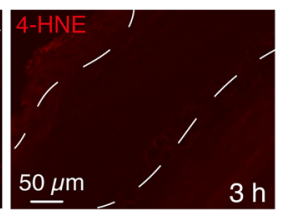

C
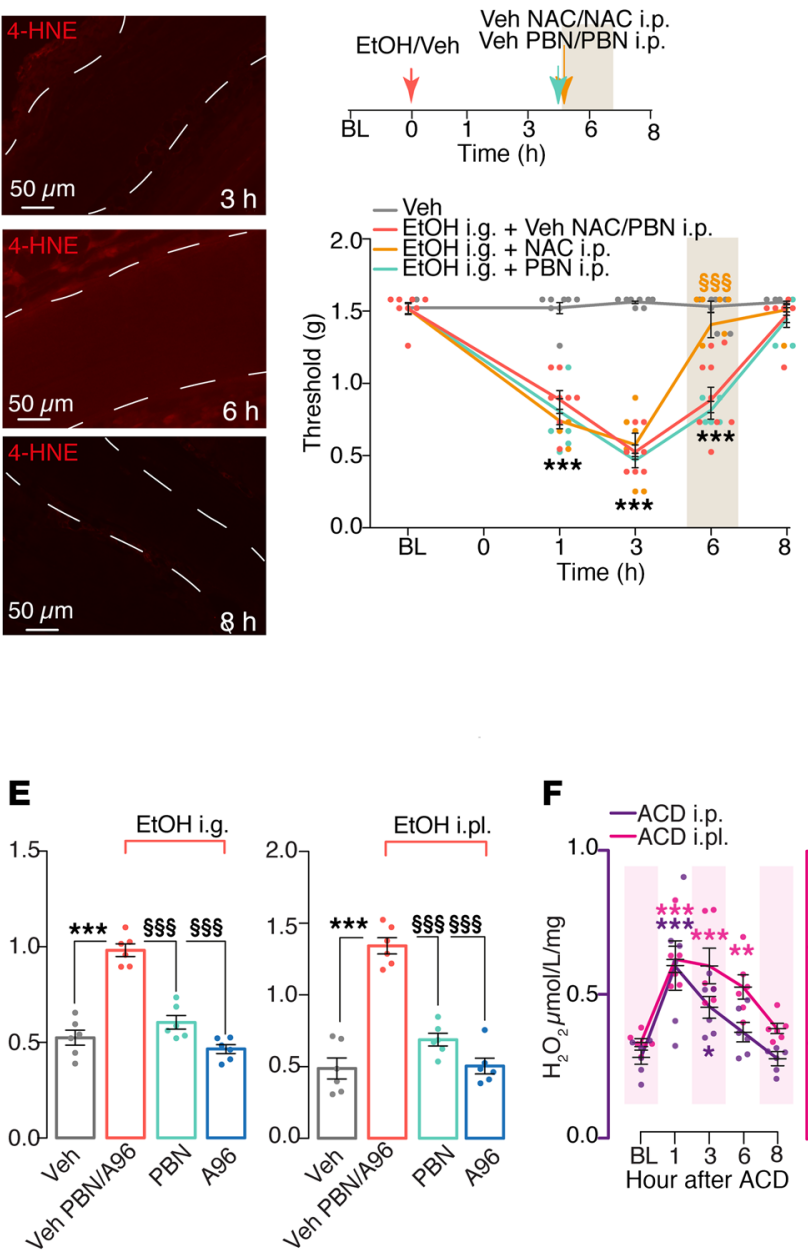

$\mathbf{F}$

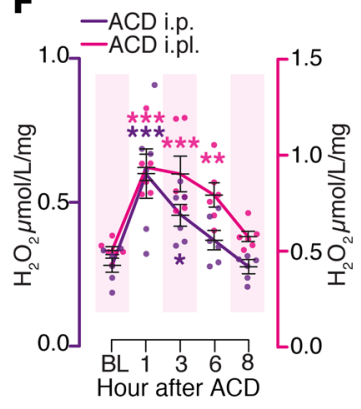

G
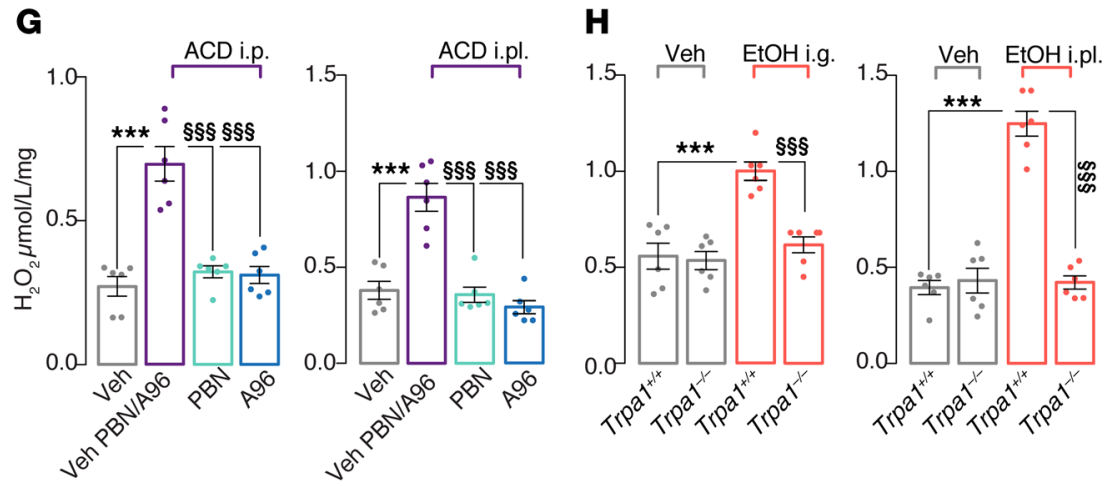

I

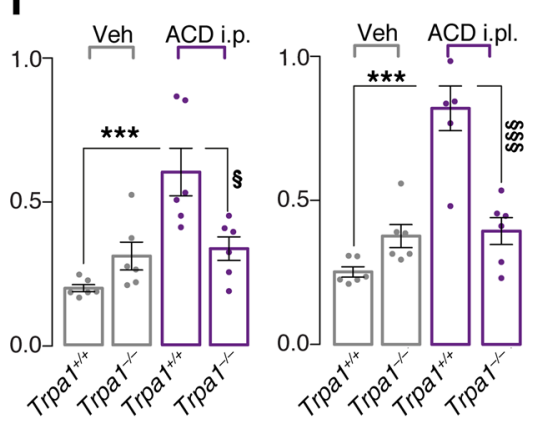

Figure 5. Acetaldehyde via TRPA1 generates ROS that sustain ethanol-evoked allodynia. (A) $\mathrm{H}_{2} \mathrm{O}_{2}$ levels (hind paw) after ethanol (EtOH, i.g. or i.pl.). (B) 4-HNE staining (plantar nerve) and levels (hind paw) after i.g. EtOH. (C) Mechanical allodynia evoked by EtOH (i.g.) or vehicle (Veh) after $\mathrm{N}$-acetylcysteine (NAC; $250 \mathrm{mg} / \mathrm{kg}$, i.p.) and PBN (50 mg/kg, i.p.) or their Veh. (D and E) $\mathrm{H}_{2} \mathrm{O}_{2}$ levels (hind paw) after i.g. and i.pl. EtOH or Veh in mice pretreated with 4-Mp (50 mg/kg, i.g.) (D), A967079 (A96, 100 mg/kg, i.p.), and PBN (100 mg/kg, i.p.) (E) or their Veh. (F) $\mathrm{H}_{2} \mathrm{O}_{2}$ levels (hind paw) after acetaldehyde (ACD; $0.1 \mathrm{mg} / \mathrm{kg}$, i.p., or $10 \mathrm{nmol}$, i.pl.). (G) $\mathrm{H}_{2} \mathrm{O}_{2}$ levels (hind-paw tissue) after ACD (0.1 mg/kg, i.p., or $10 \mathrm{nmol}$, i.pl.) or Veh and pretreatment with A96 (100 mg/kg, i.p.) or PBN (100 $\mathrm{mg} / \mathrm{kg}$, i.p.) or their Veh. (H) $\mathrm{H}_{2} \mathrm{O}_{2}$ levels (hind paw) in Trpa1 ${ }^{+/+}$and Trpa1 ${ }^{-/-}$mice treated with i.g. and i.pl. EtOH (H), ACD (0.1 mg/kg, i.p., and 10 nmol, i.pl.) (I), or their Veh. BL, baseline. Veh is the vehicle of EtOH and ACD. Where not indicated, mice are C57BL/6) and EtOH doses are: i.g., 15\%, 4 mL/kg, and i.pl., 30\%, $20 \mu \mathrm{L}$. (B, 4-HNE mean gray value) Box plots with horizontal lines at the 25th percentile, the median, and the 75th percentile and vertical lines that extend to the minimum and maximum values; all other data are mean \pm SEM with individual data points overlaid; $n=6-8$ mice for each experimental condition. ${ }^{*} P<0.05$, ${ }^{* *} P<0.01$, ${ }^{* *} P<0.001$ vs. Veh, ACD-BL, and $\mathrm{H}_{2} \mathrm{O}_{2}-\mathrm{BL}$; ${ }^{\S} P<0.05, \$ \S \S<0.001$ vs. EtOH, EtOH-A96, EtOH-PBN, EtOH-Trpa1+/+, ACD-A96, ACD-PBN, and ACD-Trpa1+/+; 1-way (A, B, and D-I) or 2-way (C) ANOVA with Bonferroni post hoc correction. 

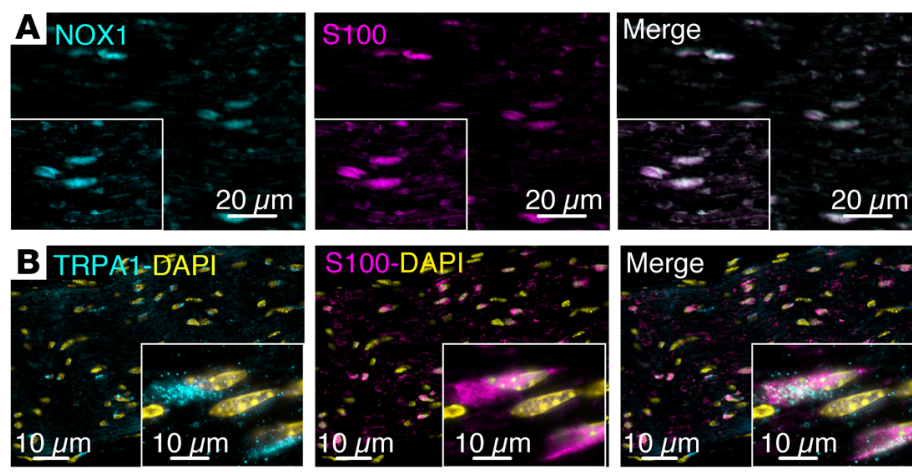

E
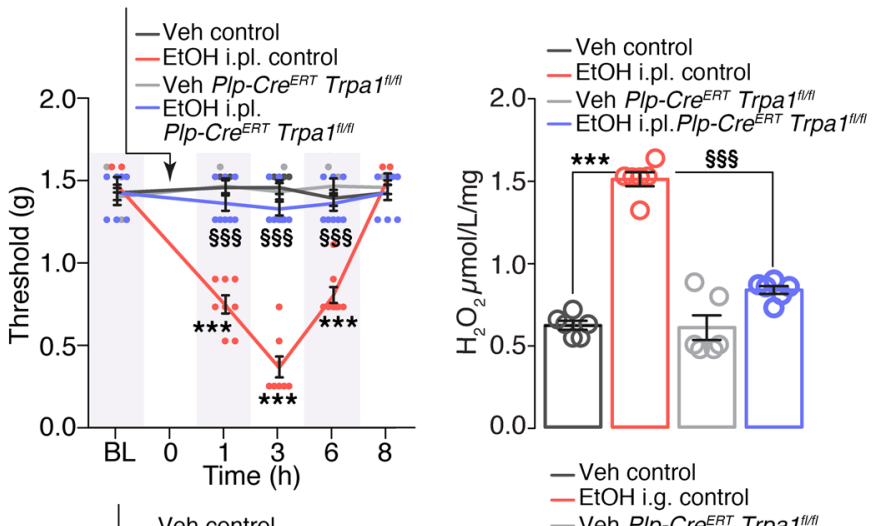

$\mathbf{F}$
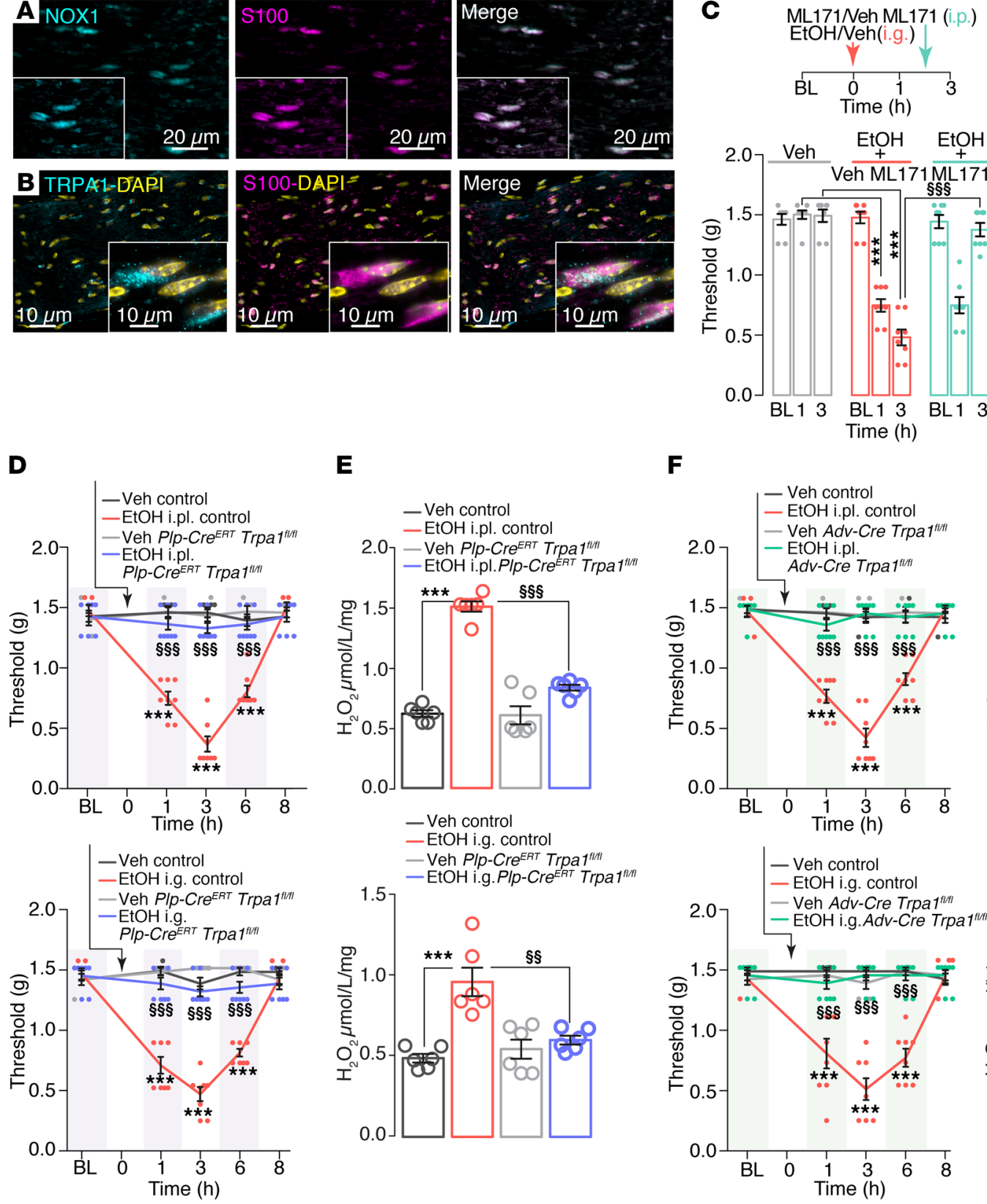

ML171Neh ML171 (i.p.) ACD/Neh (i.p.)
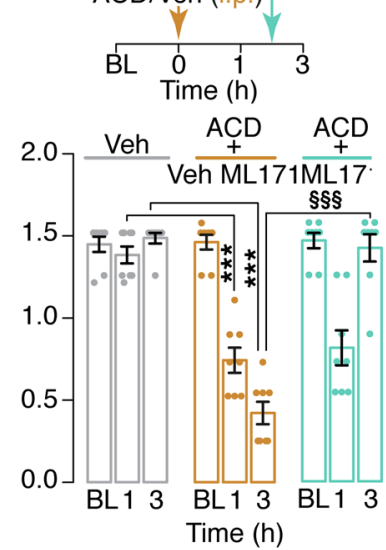

G

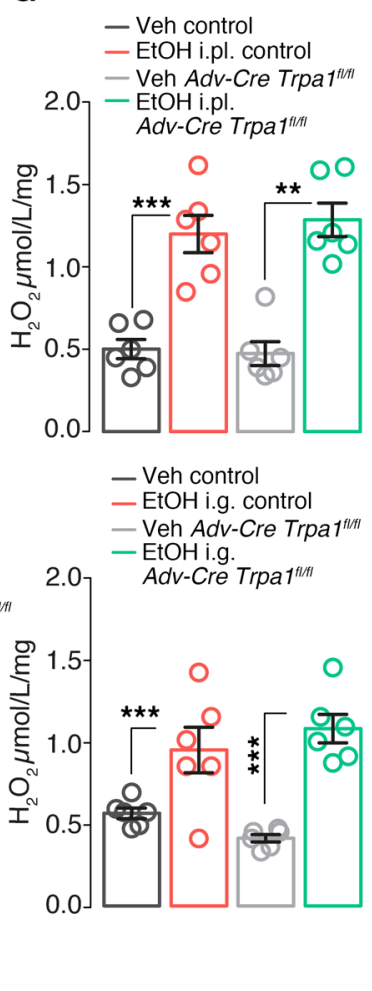

Figure 6. Schwann cell TRPA1 contributes to oxidative stress generation. (A and B) Representative images of NADPH oxidase-1 (NOX1), S100, and TRPA1 expression in plantar nerve of [57BL/6J mice. (C) Mechanical allodynia evoked by ethanol (EtOH; 15\%, 4 mL/kg, i.g.) and acetaldehyde (ACD; 0.1 mg/kg, i.p.) or their vehicle (Veh) after ML171 (100 mg/kg, i.p.) or Veh ML171 in C57BL/6) mice. (D) Time-dependent mechanical allodynia evoked by EtOH (30\%, i.pl., and $15 \%, 4 \mathrm{~mL} / \mathrm{kg}$, i.g.) or Veh in Plp-Cre ${ }^{E R T}$ Trpa fl/fl and control mice. (E) $\mathrm{H}_{2} \mathrm{O}_{2}$ levels (hind paw) after EtOH (30\%, i.pl., and 15\%, 4 mL/kg, i.g.) or Veh in Plp-Cre Trpa ${ }^{f l / f l}$ and control mice. (F) Time-dependent mechanical allodynia evoked by EtOH (30\%, i.pl., and 15\%, 4 mL/kg, i.g.) or Veh in Adv-Cre Trpa fflfl and control mice. (C) $\mathrm{H}_{2} \mathrm{O}_{2}$ levels (hind-paw tissue) after EtOH (30\%, i.pl., and 15\%, $4 \mathrm{~mL} / \mathrm{kg}$, i.g.) or Veh in Adv-Cre Trpa ${ }^{f l / f l}$ and control mice. BL, baseline. Veh is the vehicle of EtOH and ACD. Dashed lines indicate perineurium. Data are mean \pm SEM with individual data points overlaid; $n=6-8$ mice for each experimental condition. ${ }^{* *} P<0.01$, ${ }^{* *} P<0.001$ vs. Veh; ${ }^{\S \S P}<0.01$, ${ }^{\S \S} P<0.001$ vs. EtOH i.g. or EtOH i.pl. control; 1 -way (E and G) or 2 -way (C, D, and F) ANOVA with Bonferroni post hoc correction.

reversed allodynia evoked by ethanol (intragastric) and acetaldehyde (i.p.) (Figure 6C).

Our results suggest that $\mathrm{ADH}$ in the liver or in Schwann cells of the sciatic nerve converts ethanol to acetaldehyde; Schwann cell-derived acetaldehyde might activate TRPA1 in an autocrine manner to generate ROS and RCS, which could amplify and sustain allodynia. To specifically define the role of Schwann cell TRPA1 in ethanol-evoked nociception, we studied mice with
Schwann cell-specific deletion of TRPA1. Mice with conditional ablation of TRPA1 in Schwann cells (Plp1-Cre $E^{E R T} \operatorname{Trpa1}^{f / f t}$ ) (22) exhibited a normal acute nociception after intraplantar ethanol or allyl isothiocyanate (AITC; a TRPA1 agonist) (Supplemental Figure 4A). As these 2 responses are mediated by neuronal TRPV1 and TRPA1, respectively, the results indicate that the neuronal channel is unaffected by deletion of Schwann cell TRPA1. However, allodynia and generation of $\mathrm{H}_{2} \mathrm{O}_{2}$ in the paw evoked by 
A Day 0 EtOH-fed group: Euthanize

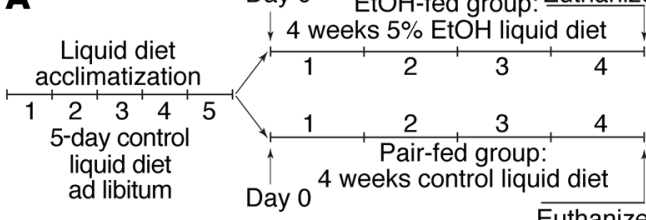

Euthanize

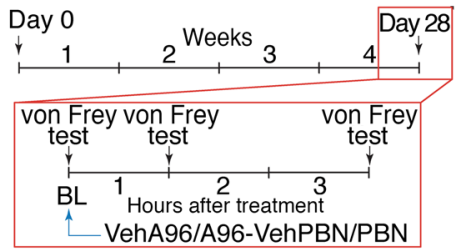

-Pair-fed control -Pair-fed Plp-Cre ERT Trpat flHI
-EtOH-fed control -EtOH-fed Plp-Cre

B
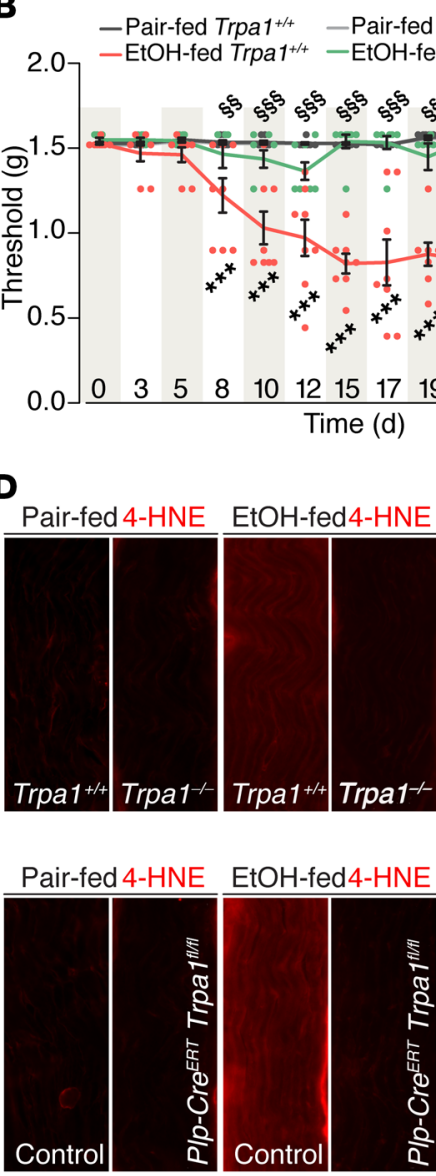

E

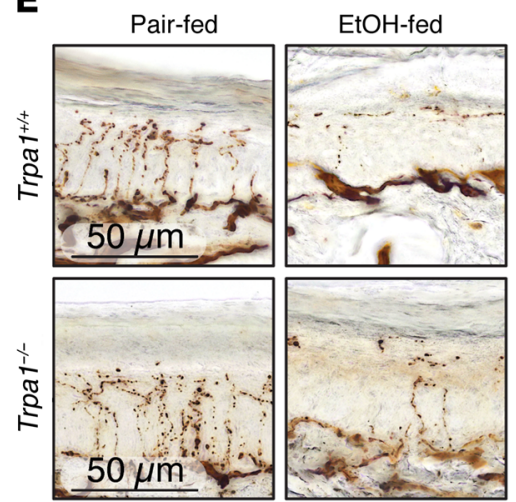

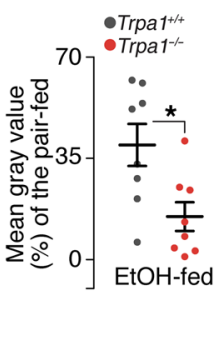
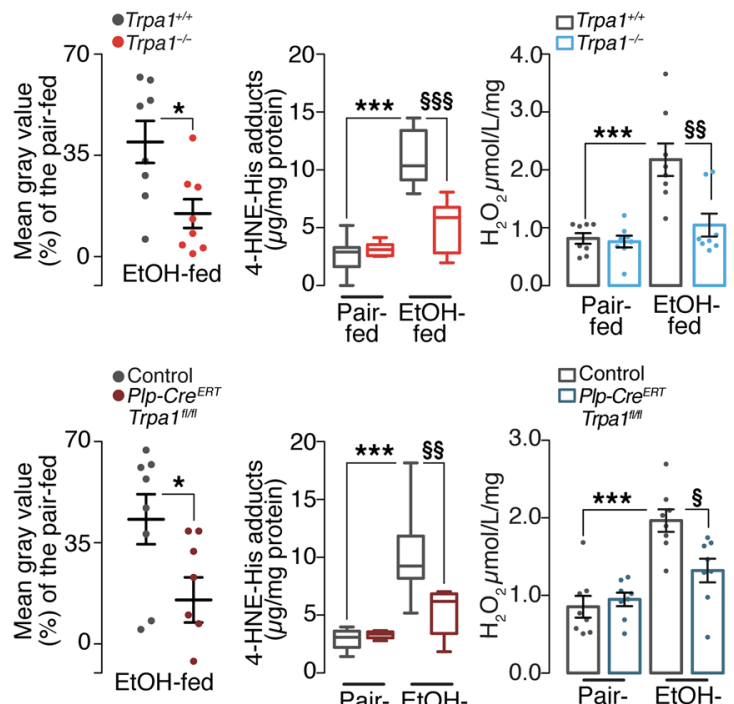

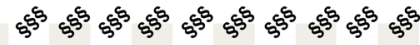

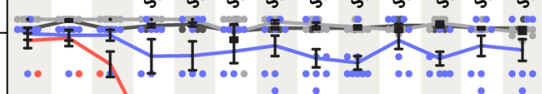<smiles>CCC(C)C(C)C</smiles>

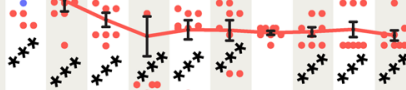

$\begin{array}{lllllllllllll}0 & 3 & 5 & 8 & 10 & 12 & 15 & 17 & 19 & 20 & 23 & 25 & 28\end{array}$ Time (d)
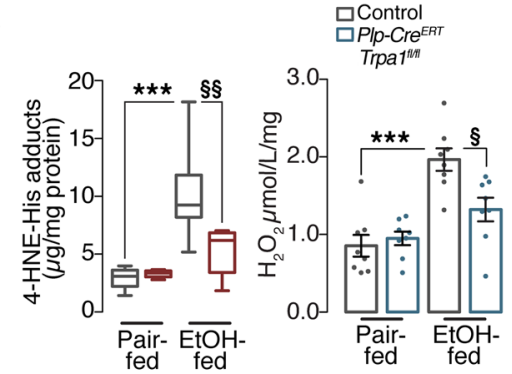
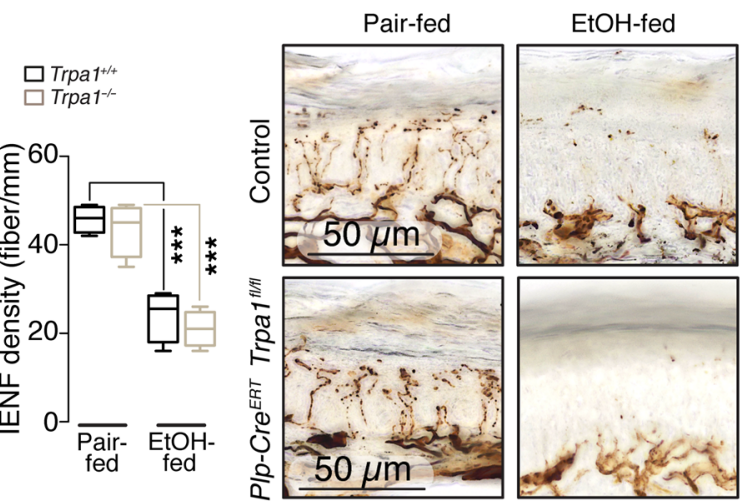
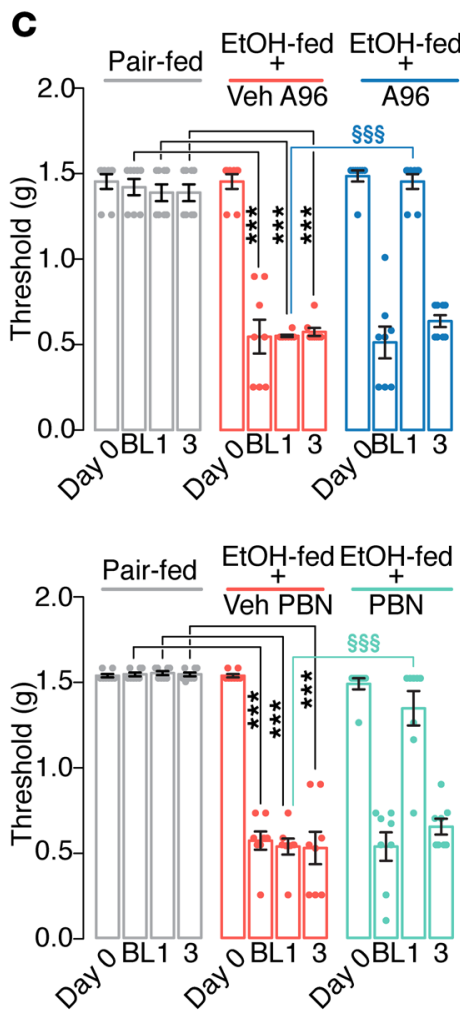

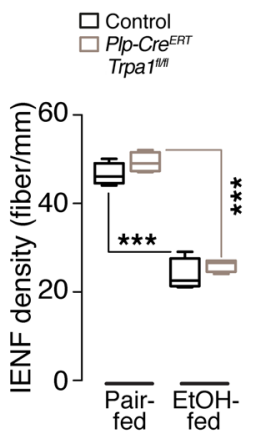


Figure 7. Schwann cell TRPA1 mediates mechanical allodynia evoked by chronic ethanol treatment. (A) Overview of the experimental design of the chronic ethanol (EtOH) feeding model. (B) Time-dependent mechanical allodynia evoked by EtOH diet (5\% [vol/vol] for 28 days) or control diet (Pair-fed) in Trpa ${ }^{+/+}$and Trpa $1^{-/-}$mice and PIp-Cre ${ }^{E R T}$ Trpaf $f^{f / / f l}$ and control mice. (C) Mechanical allodynia evoked by EtOH or control diet at day 28 after $A 967079$ (A96, $100 \mathrm{mg} / \mathrm{kg}$, i.p.) and PBN (100 mg/kg, i.p.) or their vehicle (Veh) in C57BL/6] mice. (D) Representative photomicrographs and cumulative data of 4-HNE staining (plantar nerve) and levels (hind

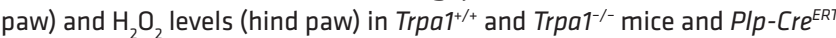
Trpa $f^{f / f l}$ and control mice at day 28 after EtOH or control diet. Original magnification $\times 20$. (E) Representative photomicrograph of PGP9.5 staining and cumulative data of intraepidermal nerve fiber (IENF) density in the

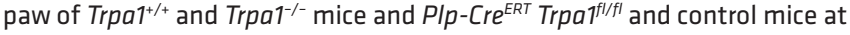
day 28 after EtOH or control diet. BL, baseline. (D, 4-HNE-His, and E) Box plots with horizontal lines at the 25th percentile, the median, and the 75th percentile and vertical lines that extend to the minimum and maximum values; all other data are mean \pm SEM with individual data points overlaid; $n=8$ mice for each experimental condition. ${ }^{*} P<0.05$, ${ }^{* * *} P<0.001$ vs. pair-fed Trpa1 ${ }^{+/+}$, pair-fed control, or EtOH-fed Veh A96/A96 day 0 ; ${ }^{\S} P<$ $0.05, \$ \S P<0.01, \$ \S \S P<0.001$ vs. EtOH-fed Trpa $1^{+/+}$, EtOH-fed control, or EtOH-fed Veh A96; unpaired 2-tailed Student's $t$ test (D, mean gray value), 1-way (D, 4-HNE-His adducts and $\mathrm{H}_{2} \mathrm{O}_{2}$, and $\mathbf{E}$ ) or 2-way (B and $\mathbf{C}$ ) ANOVA with Bonferroni post hoc correction.

intraplantar or intragastric ethanol were attenuated in Plp1-Cre $e^{E R T}$ Trpa1 $1^{f / f}$ mice (Figure 6, D and E).

To further support the involvement of Schwann cell TRPA1 in ethanol-evoked mechanical allodynia, mice were locally treated with the TRPA1 antisense oligonucleotide (TRPA1 AS-ODN) or its mismatched (TRPA1 MM-ODN) analog (both intraplantar) (Supplemental Figure 4C) or with the activated form of tamoxifen (4-hydroxytamoxifen, 4-OHT) (intraplantar) (Supplemental Figure $4 G)$. Both treatments were given by injection in the vicinity of the plantar nerve trunk, before ethanol (intraplantar or intragastric). In mice treated with TRPA1 AS-ODN or 4-OHT, acute nociception responses by intraplantar ethanol or AITC (dependent on neuronal TRPV1 and TRPA1, respectively) were preserved (Supplemental Figure 4, E and I), indicating that neuronal TRPV1 and TRPA1 were unaffected. However, colocalization of TRPA1 with the Schwann cell marker S-100 in the plantar nerve was markedly reduced (Supplemental Figure 4, D and H), confirming selective deletion of TRPA1 in Schwann cells. After intragastric ethanol, mechanical allodynia was attenuated solely in the paw treated with the TRPA1 AS-ODN, while it was unaffected in the TRPA1 MM-ODN-treated paw (Supplemental Figure 4F). Similar results were obtained with local treatment with 4-OHT. Notably, 4-OHT attenuated mechanical allodynia evoked by intragastric ethanol only in the paw treated with 4-OHT, but not in the contralateral paw (Supplemental Figure 4J). These data support the hypothesis that ethanol-evoked allodynia is initiated and maintained by Schwann cell TRPA1.

To specifically investigate the contribution of neuronal TRPA1, we studied mice with selective deletion of TRPA1 in primary sensory neurons ( $A d v$-Cre Trpa1 $\left.{ }^{f / f l}\right)$ (31). AITC-induced nociception was attenuated in $A d v$-Cre Trpa ${ }^{f / f l}$ mice, which confirms deletion of the functional channel (Supplemental Figure 4B). In Adv-Cre Trpa1 $1^{f / f}$ mice, ethanol-induced acute nociception was preserved (Supplemental Figure 4B). However, whereas allodynia evoked by intragastric or intraplantar ethanol was attenuated, $\mathrm{H}_{2} \mathrm{O}_{2}$ for- mation was unaffected (Figure 6, F and G). Thus, in contrast with the Schwann cell channel, TRPA1 expressed by nociceptor signals pain, but does not contribute to oxidative stress generation.

Chronic ethanol ingestion evokes ROS formation and allodynia dependent on Schwann cell TRPA1. In WT mice, daily ingestion of a diet containing ethanol (5\% for 28 days) (32) induced a progressively increasing mechanical allodynia that was apparent at 5-8 days, stabilized after 2 weeks, and was maintained for the entire period of observation (Figure 7, A and B). Both Trpa1 ${ }^{-/}$and Plp1-Cre ${ }^{E R T} \operatorname{Trpa}^{f / / f l}$ mice were protected from the development of allodynia (Figure 7B). At day 28 after the initiation of the ethanol diet, the allodynia observed in WT mice was reversed by treatment (i.p.) with the TRPA1 antagonist A967079 or with the ROS scavenger PBN (Figure 7C). At day 28, WT mice showed increased levels of $\mathrm{H}_{2} \mathrm{O}_{2}$ and 4-HNE in the plantar nerve that were attenuated in Trpa1 ${ }^{-/}$and Plp1-Cre ${ }^{E R T} \mathrm{Trpa}^{\mathrm{fl} / \mathrm{fl}}$ mice (Figure 7D). However, the loss of PGP9.5-positive intraepidermal nerve fibers (IENFs) observed at day 28 was unaffected in either Trpa1 ${ }^{-1-}$ or Plp1-Cre ${ }^{E R T} \operatorname{Trpa}^{7 / / f l}$ mice (Figure 7E). At day 28, the levels of ethanol or acetaldehyde in the paw of Trpa1 ${ }^{-/}$and Plp1-Cre ERT $\operatorname{Trpa}^{f / f l}$ mice were not different from those measured in respective WT and control mice (Supplemental Figure 5, A-D).

Ethanol evokes $A D H$ - and TRPA1-dependent ROS formation by human Schwann cells. Our findings reveal an unexpected but critical role for Schwann cells in ethanol-evoked mechanical allodynia in mice. To assess whether these findings could translate to humans, we studied primary cultures of human Schwann cells (HSCs) and Schwann cells in sections of human skin. HSCs in culture expressed immunoreactive $\mathrm{S} 100$ and immunoreactive ADH (Figure 8A). In nerve bundles in biopsies of human skin, immunoreactive ADH colocalized with $\mathrm{S} 100$ (Figure $8 \mathrm{~B}$ and Supplemental Figure 2B). mRNA encoding multiple isoforms of $\mathrm{ADH}$ (1A, 1B, 1C, and 5) was amplified from HSCs in culture (Figure 8C). Exposure of HSCs to ethanol caused a concentration-dependent generation of acetaldehyde that was inhibited by 4-Mp, and thus depends on ADH activity (Figure 8D). S100-positive HSCs in culture (Figure $8 \mathrm{E}$ ) and in sections of human skin also expressed immunoreactive TRPA1 (Figure 8F and Supplemental Figure 2B). Cultured HSCs expressed TRPA1 mRNA (Figure 8G). Exposure of HSCs to AITC, acetaldehyde, or $\mathrm{H}_{2} \mathrm{O}_{2}$ elicited increases in intracellular calcium that were attenuated by A967079 (Figure 8H). Prolonged exposure of HSCs to ethanol induced a delayed and sustained increase in calcium responses, which was attenuated by pretreatment with 4-Mp or A967079 (Figure 8I). S100-positive HSCs in culture and in sections of human skin also expressed immunoreactive NOX1 (Figure 8, J and $\mathrm{K}$, and Supplemental Figure 2B). In addition, cultured HSCs expressed NOX1 mRNA (Figure 8L). AITC, acetaldehyde, or $\mathrm{H}_{2} \mathrm{O}_{2}$ elicited calcium-dependent increases in $\mathrm{H}_{2} \mathrm{O}_{2}$ in HSCs that were reduced by A967079 (Figure 8M).

\section{Discussion}

Our results show that intraplantar injection of ethanol in the mouse hind paw causes 2 sensory responses, which are temporally and mechanistically distinct. After an immediate and short-lived (5 minutes) nociceptive response, mice exhibited a delayed and prolonged (1-6 hours) mechanical allodynia. TRPV1 antagonism or deletion prevented the immediate nociceptive response, which 

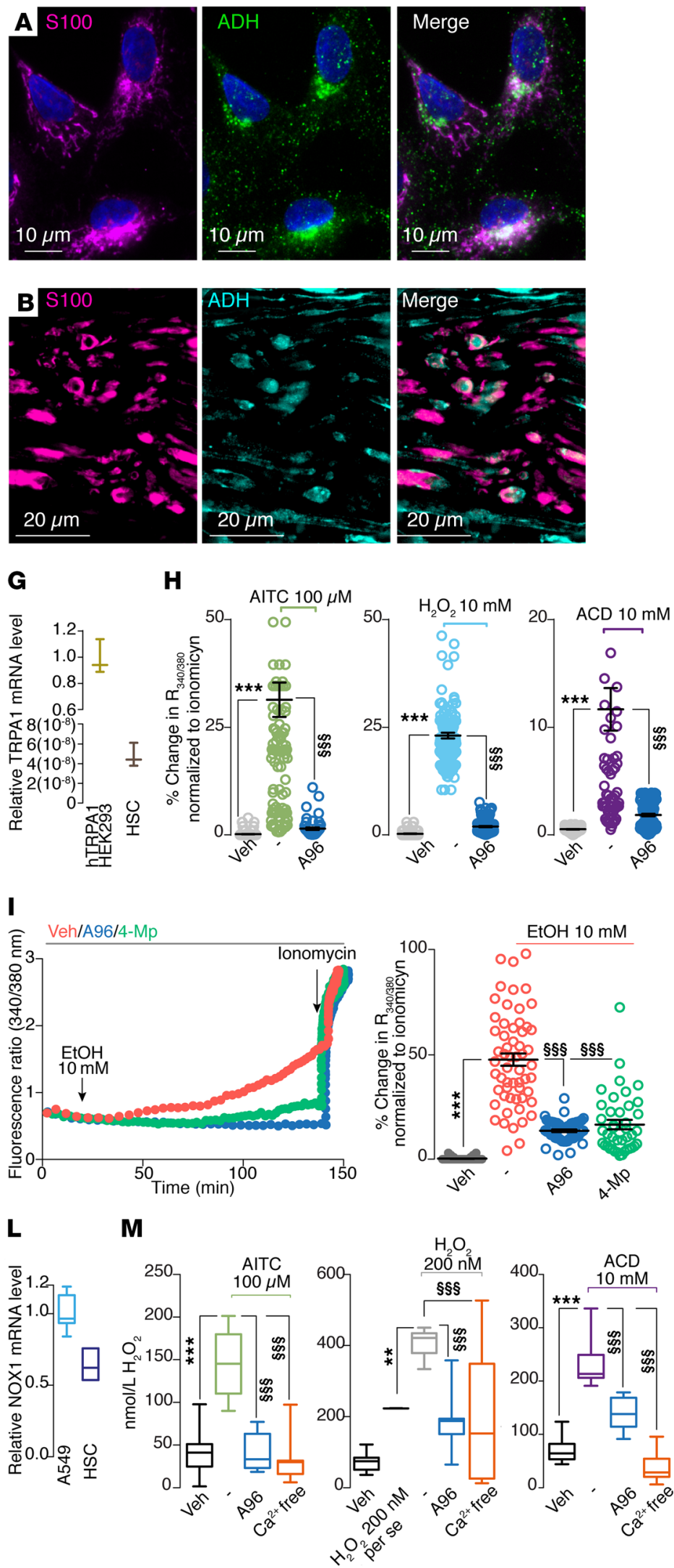

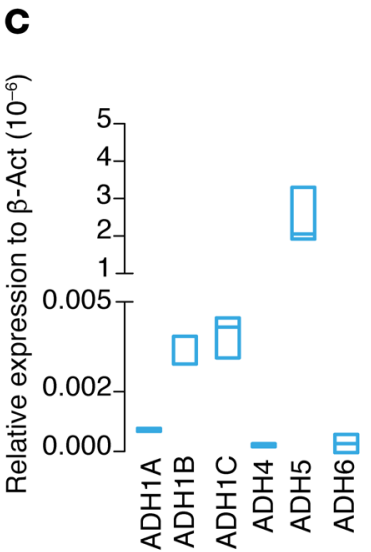

D
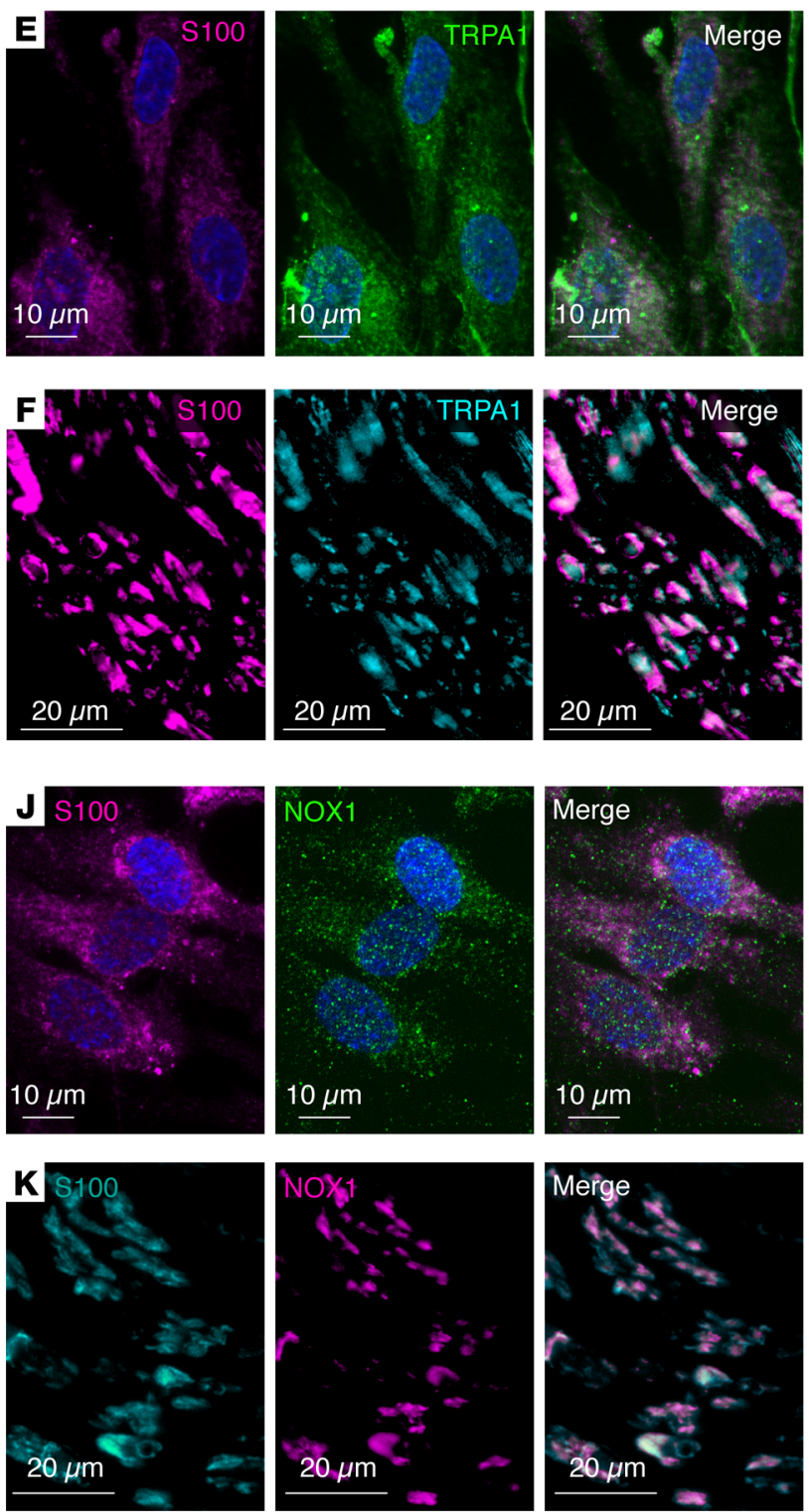
Figure 8. TRPA1 and ADH are present in human Schwann cells. (A, B, E, $\mathbf{F}, \mathbf{J}$, and $\mathbf{K}$ ) Representative images of $\mathrm{S} 100$ and ADH expression (A and B), TRPA1 (E and $\mathbf{F}$ ), and NOX1 immunofluorescence (J and $\mathbf{K}$ ) in cultured human Schwann cells (HSCs) and human skin. (C) ADH1A, ADH1B, ADH1C, ADH4, ADH5, and ADH7 mRNA relative expression in HSCs. (D) Dosedependent acetaldehyde (ACD) levels in HSCs exposed to ethanol (EtOH; 1-100 mM) and treated with 4-Mp (100 $\mu \mathrm{M})$. (C) TRPA1 mRNA expression in HSCs relative to human TRPA1-HEK293 cells. (H) Intracellular calcium $\left(\left[\mathrm{Ca}^{2+}\right]\right.$ ) response to allyl isothiocyanate (AITC; $\left.100 \mu \mathrm{M}\right), \mathrm{H}_{2} \mathrm{O}_{2}(10 \mathrm{mM})$, and ACD (10 mM) in HSCs in the presence of A967079 (A96, $30 \mu \mathrm{M})$ or its vehicle (Veh). (I) $\left[\mathrm{Ca}^{2+}\right]_{\mathrm{i}}$ response to EtOH $(10 \mathrm{mM})$ in HSCs in the presence of A96 $(30 \mu \mathrm{M}), 4-\mathrm{Mp}(100 \mu \mathrm{M})$, or their Veh. (L) NOX1 mRNA expression in $\mathrm{HSCs}$ relative to $\mathrm{A} 549$ cells. (M) $\mathrm{H}_{2} \mathrm{O}_{2}$ release evoked by AITC $(100 \mu \mathrm{M}), \mathrm{H}_{2} \mathrm{O}_{2}$ $(200 \mathrm{nM})$, and ACD $(10 \mathrm{mM})$ in HSCs in the presence of $A 96(30 \mu \mathrm{M})$ or Veh or in $\mathrm{Ca}^{2+}$-free medium. Veh is the vehicle of $\mathrm{EtOH}, \mathrm{AITC}, \mathrm{H}_{2} \mathrm{O}_{2}$, and ACD. (C, D, G, L, and $\mathbf{M}$ ) Box plots with horizontal lines at the 25th percentile, the median, and the 75th percentile and vertical lines that extend to the minimum and maximum values; $n=3$ replicates from 2 independent experiments in $\mathbf{C}, \mathbf{G}$, and $\mathbf{L}$, and $n=8$ replicates from 3 independent experiments in $\mathbf{D}$ and $\mathbf{M}$; ( $\mathbf{H}$ and $\mathbf{I})$ data are mean \pm SEM with individual data points overlaid, 41 to 327 cells; $n=3-5$ experiments. ${ }^{*} P<0.05$, ${ }^{* * *} P<$ 0.001 vs. Veh; ${ }^{\circledR} P<0.05, \$ \S \S<0.001$ vs. EtOH $(10 \mathrm{mM})$, AITC $(100 \mu \mathrm{M}), \mathrm{H}_{2} \mathrm{O}_{2}$ (200 nM), and ACD (10 mM); (D, H, I, and M) 1-way ANOVA with Bonferroni post hoc correction.

is thus mediated by ethanol activation of TRPV1, as previously reported (16). In contrast, TRPV1 disruption did not affect the delayed and sustained mechanical allodynia, which occurs by a different mechanism.

Genetic or pharmacological impairment of ADH that results in increased acetaldehyde levels enhances the susceptibility of affected individuals to alcohol-evoked symptoms $(13,14)$. Our observations that pretreatment with the ADH inhibitor 4-Mp prevented the allodynia evoked by intraplantar ethanol and that intraplantar injection of acetaldehyde caused allodynia similar to that evoked by ethanol support the hypothesis that acetaldehyde mediates the prolonged allodynic effect of ethanol. The findings that genetic deletion or pharmacological antagonism of TRPA1 prevented ethanol- and acetaldehyde-evoked allodynia are consistent with the hypothesis that the aldehyde and not its precursor gates TRPA1 (20) and plays a major role in the allodynia that mimics the neuropathic pain observed in alcoholics. In contrast, deletion of TRPV4, a proalgesic channel expressed by a subset of nociceptors, did not affect either acute nociception or allodynia. Thus, while acute nociception is due to a direct action of ethanol on TRPV1, delayed and sustained allodynia is mediated by TRPA1, via ADH-dependent generation of acetaldehyde.

We investigated the contribution of TRPA1 to pain after oral administration of ethanol, which more closely resembles the situation in alcoholics. In contrast to intraplantar administration, intragastric administration of ethanol did not cause acute painrelated behavior. However, intragastric ethanol did cause delayed and sustained allodynia in WT but not Trpa1 ${ }^{--}$mice; deletion of either TRPV1 or TRPV4 did not affect allodynia. The ability of intraperitoneal acetaldehyde to mimic the response evoked by intragastric ethanol implicates this metabolite as a mediator of TRPA1-dependent allodynia. Although the major site of alcohol metabolism is the liver, where the bulk of acetaldehyde is generated $(25,26)$, several extrahepatic cells express $\operatorname{ADH}(23,24)$. The $\mathrm{ADH}$ inhibitor 4-Mp, when given locally (intraplantar adminis- tration), prevented acetaldehyde increase and allodynia evoked by local (intraplantar) ethanol and, although partially, reduced increases in acetaldehyde and allodynia elicited by intragastric ethanol. Complete inhibition of allodynia and increases in acetaldehyde levels evoked by intragastric ethanol were, however, obtained by systemic 4-Mp administration. These results suggest that acetaldehyde, which mediates allodynia, derives in part from the systemic circulation, and in part from local production within the paw tissue. ADH mRNA and protein were confined to Schwann cells that ensheath sensory nerve fibers in the paw. The ability of the paw tissue and cultured mouse Schwann cells to convert ethanol into acetaldehyde in a 4-Mp-sensitive manner supports the proposal that Schwann cells are the local source of acetaldehyde, which engages TRPA1 to mediate allodynia.

Whereas the TRPA1 antagonist A967079 inhibited ethanolevoked allodynia when administered before or after ethanol, the $\mathrm{ADH}$ inhibitor 4-Mp prevented allodynia only when given before ethanol. This finding suggests that, whereas TRPA1 engagement is constantly required, acetaldehyde is necessary to initiate, but is not sufficient to maintain, allodynia. The transient (1-hour duration) increase in acetaldehyde levels in the paw, compared with the sustained allodynia (6 hours), strengthens the view that the prolonged allodynia is not maintained by acetaldehyde. Acetaldehyde can generate ROS $(27,29)$, which are among the several endogenous ligands of TRPA1 (17-19). $\mathrm{H}_{2} \mathrm{O}_{2}$ levels peaked in paw homogenates 1-3 hours, and returned to baseline 6 hours, after ethanol. However, in the mouse paw, immunostaining for the carbonylic by-product of oxidative stress 4-HNE (33), a known TRPA1 activator (21), increased 3 hours, and peaked 6 hours, after ethanol, when allodynia was still robustly present. In agreement with the time courses of $\mathrm{H}_{2} \mathrm{O}_{2}$ and 4-HNE levels in the mouse paw, treatment with the ROS scavenger PBN attenuated allodynia 3 hours, but not 6 hours, after ethanol, while the aldehyde scavenger NAC was effective 6 hours after ethanol. These findings suggest that TRPA1 engagement by mediators efficiently scavenged by PBN does not outlast approximately 4 hours after exposure to ethanol, whereas attenuation of allodynia 6 hours after ethanol is provided exclusively by compounds able to scavenge aldehydic compounds, such as 4-HNE. The ability of the ROS scavenger PBN to attenuate paw allodynia indicates that oxidative stress sustains allodynia and raises the question of how ethanol and acetaldehyde generate ROS. We recently reported in a mouse model of sciatic nerve injury that ROS, which sustain mechanical allodynia, are produced via the TRPA1/NOX1 pathway in Schwann cells ensheathing sciatic nerve fibers (22). Therefore, we investigated the possibility that a similar mechanism contributes to ethanol-evoked allodynia. In support of this hypothesis, Plp1-Cre ${ }^{E R T} \operatorname{Trpa1}^{f / f l}$ mice, which selectively lack TRPA1 in Schwann cells, showed attenuated allodynia and reduced $\mathrm{H}_{2} \mathrm{O}_{2}$ levels in paw tissue. NOX1 has been reported as the major enzyme isoform responsible for the TRPA1-dependent oxidative burst in Schwann cells (22). We confirmed this finding, since the selective NOX1 inhibitor ML171 (34) attenuated ethanol-evoked allodynia.

Finally, we asked whether the TRPA1 that mediates ethanolevoked allodynia is exclusively the channel expressed by Schwann cells, or whether the neuronal channel might also contribute. Mice with selective deletion of TRPA1 in primary sensory neu- 
rons, Adv-Cre Trpa1 $1^{f / f l}$ mice (31), exhibited a markedly attenuated ethanol-evoked paw allodynia. However, the increased levels of $\mathrm{H}_{2} \mathrm{O}_{2}$ in the paw tissue were unaffected. These observations indicate that the Schwann cell TRPA1/NOX1 pathway is necessary to maintain the prolonged oxidative stress generation required to target the neuronal TRPA1, which eventually sustains allodynia. Plp1-Cre ${ }^{E R T} \operatorname{Trpa1}^{\text {fl/fl }}$ mice are selectively deficient in TRPA1 in the Schwann cell and oligodendrocyte lineage. Thus, the implication of oligodendrocytes in the brain signaling of allodynia evoked by intragastric ethanol should be considered. However, several observations suggest that initiation and maintenance of paw allodynia are promoted by a series of local events driven by TRPA1 of Schwann cell ensheathing the plantar nerve. Plp1-Cre $e^{E R T}$ $\operatorname{Trpa1}^{f l / f l}$ mice not only failed to produce allodynia, but were unable to generate the local oxidative stress response that is required to sustain allodynia. Local treatment with a TRPA1 AS-ODN or with the active tamoxifen metabolite 4-OHT, which directly binds to the tamoxifen-inducible estrogen receptor, attenuated intragastricethanol-evoked allodynia only in the treated paw, without affecting the pain response in the contralateral side. These findings imply that the drugs did not diffuse to distant sites, including the brain, but maintained their action confined to the treated paw. Thus, the most parsimonious hypothesis is that Schwann cell TRPA1, by promoting local oxidative stress generation, is necessary and sufficient to sustain ethanol-evoked allodynia. Nonetheless, these observations do not exclude a contribution of oligodendrocyte TRPA1 to the central processing of the pain response. The generation of mice lacking TRPA1 solely in Schwann cells would be required to definitively determine the selective role of TRPA1 in this cell type.

Painful neuropathy is produced by chronic alcohol ingestion and is associated with peculiar pathological features, such as the loss of IENFs (5). We reproduced a chronic condition in mice by feeding them an ethanol-containing diet. The observation that both Trpa1 $1^{-/}$and Plp1-Cre ERT Trpa1 ${ }^{l / / f l}$ mice did not develop allodynia indicated that TRPA1, and specifically the channel expressed by Schwann cells, is necessary and sufficient to generate the painful condition. Reduced levels of either $\mathrm{H}_{2} \mathrm{O}_{2}$ or 4-HNE in the plantar nerve of Trpa1 ${ }^{-/}$and Plp1-Cre ${ }^{E R T}$ Trpa1 $1^{f / f l}$ mice and the ability of the TRPA1 antagonist and the ROS scavenger to reverse allodynia suggest that chronic ethanol ingestion activates a proalgesic mechanism identical to that induced by acute ethanol exposure. Thus, the oxidative stress that signals pain is generated by a sustained gating of Schwann cell TRPA1 by acetaldehyde generated by the daily ethanol ingestion.

Our results show that in mice receiving chronic ethanol administration deletion of Schwann cell TRPA1 does not ameliorate IENF loss, but does attenuate allodynia. Thus, Schwann cell TRPA1 and the ensuing oxidative stress burst are not crucial for the neuropathy, and there is no stringent correspondence between IENF degeneration and neuropathic pain. After the loss of IENFs, the remaining extraepithelial nerve fibers with their ensheathing Schwann cells are sufficient to elicit mechanical allodynia. Various interventions, including the immunomodulator minocycline (35), erythropoietin (36), a poly(ADP-ribose) polymerase inhibitor (37), and a TRPA1 antagonist (38), have been reported to attenuate IENF loss and the ensuing neuropathic pain responses. A series of differences may explain the discrepancy with our current data, including the causative agent of the neuropathy, alcohol versus streptozotocin and paclitaxel, or, in the case of TRPA1, systemic treatment with an antagonist that nonselectively targets TRPA1, not solely in Schwann cells, but in all cells where it is expressed.

The presence of thiamine deficiency in heavy alcohol drinkers suggested that the beriberi neuropathy was the underlying cause of the neurological symptoms in alcoholics. However, investigation of respective clinicopathological features has clearly differentiated the two conditions (7). Alcoholic neuropathy is sensorydominant and predominantly shows loss of small fibers, while thiamine deficiency neuropathy is motor-dominant with mainly large fiber loss (7). The small-fiber polyneuropathy documented in heavy alcohol drinkers with normal thiamine status (39) further supported the direct role of alcohol and acetaldehyde, and not of thiamine deficiency, in alcoholic neuropathy (7, 39, 40). Assessment of ethanol-evoked allodynia in mice underscores the implication of TRPA1 either in acute (single administration) or chronic (prolonged exposure) settings. However, pain (with or without burning quality) is the initial major symptom of alcoholic neuropathy; it develops slowly, extending over a period of months, and is associated with allodynia and hyperalgesia (3). Thus, a limitation of the present murine study is that only one (mechanical allodynia) among the various pain modalities reported by alcoholics has been investigated.

While TRPV1 is key to evoke the acute nociceptive behavior caused by local exposure to ethanol, TRPA1 uniquely mediates the prolonged paw allodynia that models in mice one pain symptom observed in patients. However, multiple ethanol-derived mediators contribute to the TRPA1-mediated paw allodynia. Acetaldehyde generated by liver ADH (Supplemental Figure 6) and locally by Schwann cell ADH commits Schwann cell TRPA1/NOX1 to generate ROS. ROS released from Schwann cells engage TRPA1 in nociceptors initially and up to 3 hours after ethanol. The peroxidation-dependent generation of carbonylic by-products, including 4-HNE, sustains the final TRPA1 targeting. The translational value of the present findings is strengthened by the observation that human Schwann cells are fully equipped to exert all the functions found in mouse Schwann cells. Human Schwann cells express ADH, TRPA1, and NOX1 mRNA and protein, and are capable of converting ethanol into acetaldehyde. Human Schwann cells respond to ethanol (via acetaldehyde), acetaldehyde, and $\mathrm{H}_{2} \mathrm{O}_{2}$ with a TRPA1dependent calcium response and the ensuing generation of oxidative stress. Thus, inhibition of any of the steps reported here in mice may be translated to human Schwann cells and could be beneficial to attenuate pain associated with alcohol consumption in patients.

The presence of ADH in Schwann cells that express TRPA1/ NOX1 and their ability to generate oxidative stress identify an autocrine pathway that we propose as a major contributing mechanism in alcohol-induced mechanical allodynia. Moreover, this pathway may be important in other conditions produced by exposure to alcohol. The reported presence of TRPA1 in oligodendrocytes (41) suggests that similar detrimental mechanisms may operate in the central nervous system to mediate the toxic effect associated with alcohol ingestion. Further studies are required to explore whether Schwann cell TRPA1 mediates pain-like responses evoked by chronic ethanol in humans. 


\section{Methods}

\section{Animals}

The following mouse strains were used: C57BL/6J (male, 20-25 g, 5-6 weeks; Envigo); littermate WT (Trpa1 $\left.{ }^{+/+}\right)$and TRPA1-deficient mice (Trpa1 ${ }^{-/}$, B6.129P-Trpa1 ${ }^{\mathrm{tm} 1 \mathrm{Kykw}} / \mathrm{J}, 25-30 \mathrm{~g}$, 5-8 weeks; The Jackson Laboratory) (42); littermate WT $\left(\operatorname{Trpv} 4^{+/+}\right)$and TRPV4-deficient (Trpv4 $4^{-/}$) mice (25-30 g, 5-8 weeks) (43); and littermate WT (Trpv1 $1^{+/+}$) and TRPV1-deficient mice (Trpv1 1/-, B6.129X1-Trpv1 $1^{\mathrm{tm} 1 \mathrm{ul}} / \mathrm{J}, 25-30 \mathrm{~g}$, 5-8 weeks; The Jackson Laboratory). All these strains were generated by C57BL/6 background.

To selectively delete the Trpa1 gene in primary sensory neurons, 129S-Trpa1 ${ }^{\text {tm2Kykw }} / \mathrm{J}$ mice (floxed Trpa1, Trpa1 ${ }^{\text {flfl }}$, stock 008649, The Jackson Laboratory), which possess loxP sites on either side of the S5/S6 transmembrane domains of the Trpa1 gene, were crossed with hemizygous Advillin-Cre male mice (44). The progeny (Adv-Cre Trpa $\left.1^{f / f}\right)$ was genotyped by standard PCR for Trpa1 and Advillin-Cre. Mice negative for Advillin-Cre (Adv-Cre- Trpa1 $\left.1^{f / f l}\right)$ were used as control. Successful Advillin-Cre-driven deletion of TRPA1 mRNA was confirmed by quantitative reverse transcriptase PCR (RT-qPCR) (45). To generate mice in which the Trpa1 gene was conditionally silenced in Schwann cells/oligodendrocytes, homozygous 129S-Trpa1 ${ }^{\text {tm2Kykw }}$ J mice (floxed TRPA1, Trpa1 ${ }^{f / f l}$, stock 008649, The Jackson Laboratory) were crossed with hemizygous B6.Cg-Tg(Plp1-Cre $\left.{ }^{\text {ERT }}\right) 3 \mathrm{Pop} / \mathrm{J}$ mice (Plp1-Cre ${ }^{E R T}$, stock 005975, The Jackson Laboratory) (Plp1, proteolipid protein myelin 1) expressing a tamoxifen-inducible Cre in myelinating cells (22). The progeny (Plp1-Cre Trpa $1^{f / f f}$ ) was genotyped by standard PCR for Trpa1 and Plp1-Cre $e^{E R T}$ (22). Mice negative for Plp1-Cre $e^{E R T}$ (Plp1-Cre Trpa1 $\left.^{f / f l}\right)$ were used as control. Both $C r e^{E R T}$-positive and $C r e^{E R T}$-negative mice homozygous for floxed Trpa1 (Plp1-Cre ${ }^{E R T} T r p a 1^{f l / f l}$ and Plp1-Cre ${ }^{E R T-} \mathrm{Trpa1}^{f / f l}$, respectively) were treated with intraperitoneal (i.p.) tamoxifen $(1 \mathrm{mg} / 100 \mu \mathrm{L}$ in corn oil once a day for 5 consecutive days) (22), resulting in Cre-mediated ablation of Trpa1 in PLP-expressing Schwann cells/oligodendrocytes. Successful Cre-driven deletion of TRPA1 mRNA was confirmed by RT-qPCR (22). In addition, some Plp1$C r e^{E R T} \operatorname{Trpa1}^{f / f l}$ and Plp1-Cre ${ }^{E R T-} \operatorname{Trpa1}^{f l / f l}$ mice were treated with the activated form of tamoxifen (4-hydroxytamoxifen [4-OHT], intraplantar [i.pl.], $0.02 \mathrm{mg} / 10 \mu \mathrm{L}$ once a day for 4 consecutive days).

\section{Study design}

Group size of $n=8$ animals for behavioral experiments was determined by sample size estimation using $G^{*}$ Power (version 3.1) (46) to detect size effect in a post hoc test with type 1 and 2 error rates of $5 \%$ and $20 \%$, respectively. Allocation concealment of mice to vehicle(s) or treatment(s) group was performed using a randomization procedure (http://www.randomizer.org/). The assessors were blinded to the identity (genetic background or allocation to treatment group) of the animals. Identity of the animals was unmasked to assessors only after data collection. Every effort was made to minimize the discomfort and pain of the animals in each phase of the study. Mice were housed in a temperature- and humidity-controlled vivarium (12-hour dark/12-hour light cycle, free access to food and water, 8 animals per cage). Mice were acclimatized in a quiet, temperature-controlled room $\left(20^{\circ} \mathrm{C}-22^{\circ} \mathrm{C}\right)$ for 1 hour before behavioral studies that were done between 9 am and $5 \mathrm{pm}$. Animals were anesthetized with a mixture of ketamine and xylazine $(90 \mathrm{mg} / \mathrm{kg}$ and $3 \mathrm{mg} / \mathrm{kg}$, respectively, i.p.) and euthanized with inhaled $\mathrm{CO}_{2}$ plus
$10 \%-50 \% \mathrm{O}_{2}$. If not otherwise indicated, reagents were obtained from Sigma-Aldrich.

In functional and biochemical studies, ethanol was given by i.pl. (30\%) or intragastric (i.g.) $(15 \%, 4 \mathrm{~mL} / \mathrm{kg})$ administration and acetaldehyde by i.pl. (20 nmol) or i.p. $(0.1 \mathrm{mg} / \mathrm{kg})$ administration. Vehicle of i.pl. or i.g. ethanol and i.pl. or i.p. acetaldehyde was $0.9 \% \mathrm{NaCl}$. Vehicle of the various drugs was $4 \%$ DMSO and $4 \%$ Tween- 80 in $0.9 \% \mathrm{NaCl}$ for i.pl. or i.p. administration and 0.5\% carboxymethylcellulose for i.g. administration. Twenty microliters per site was injected in all i.pl. administrations. SB366791 [N-(3-methoxyphenyl)-4-chlorocinnamide] (1 $\mathrm{mg} / \mathrm{kg}$, i.p.) or vehicle was administered 30 minutes before i.pl. ethanol or vehicle. A967079 [(1E,3E)-1-(4fluorophenyl)-2-methyl-1-penten-3-one oxime] and phenyl- $\alpha$-tertbutyl nitrone (PBN) (both $100 \mathrm{mg} / \mathrm{kg}$, i.p., or $100 \mu \mathrm{g}$, i.pl.) or their vehicle were administered 1 hour after i.pl. or i.g. ethanol and i.pl. or i.p. acetaldehyde or their vehicle. PBN (100 mg/kg, i.p.) or vehicle was also given 5 hours after i.g. ethanol. 4-Methylpyrazole (4-Mp) $(50 \mathrm{mg} / \mathrm{kg}$, i.g., or $100 \mu \mathrm{g}$, i.pl.) or vehicle was administered $30 \mathrm{~min}-$ utes before i.pl. or i.g. ethanol or vehicle and 2 hours after i.g. ethanol or vehicle. $\mathrm{N}$-acetylcysteine (NAC) $(250 \mathrm{mg} / \mathrm{kg}$, i.p.) or vehicle was administered 5 hours after i.g. ethanol. ML171 (2-acetylphenothiazine; $60 \mathrm{mg} / \mathrm{kg}$, i.p.; Tocris Bioscience) or vehicle was administered 2 hours after i.g. ethanol and i.p. acetaldehyde or their vehicles.

Phosphorothioate-modified TRPA1 AS-ODN (sequence: 5'-TATCGCTCCACATTGCTAC-3') and TRPA1 MM-ODN (sequence: $5^{\prime}$-ATTCGCCTCACATTGTCAC-3') (22) (10 nmol/10 $\mu \mathrm{L}$, i.pl.) were administered once a day for 4 consecutive days. Some mice treated with TRPA1 AS/MM-ODN or with 4-OHT were tested for the acute nociceptive response to allyl isothiocyanate (AITC) $(20 \mathrm{nmol}$, i.pl.) or ethanol (30\%, i.pl.) or their vehicles (0.5\% DMSO or $0.9 \% \mathrm{NaCl}$, respectively).

Blood for ethanol assay was obtained from anesthetized mice at 15, 30, 60, and 180 minutes after i.g. ethanol. Liver and hind-paw tissues for ethanol and acetaldehyde assays were obtained from mice euthanized 15, 30, 60, and 180 minutes after i.pl. or i.g. ethanol. Some mice were treated with $4-\mathrm{Mp}(100 \mu \mathrm{g}$, i.pl., or $50 \mathrm{mg} / \mathrm{kg}$, i.g.) $30 \mathrm{~min}$ utes before or 1 hour after i.pl. or i.g. ethanol or their vehicles and euthanized 2 hours after ethanol. For $\mathrm{H}_{2} \mathrm{O}_{2}$ assay, hind-paw tissues were taken from mice euthanized before and 1, 3, 6, and 8 hours after i.pl. or i.g. ethanol and acetaldehyde or their vehicles. In additional experiments, paw tissues were taken from mice treated with A967079 (100 $\mathrm{mg} / \mathrm{kg}$, i.p.) or PBN (100 mg/kg, i.p.) or their vehicles 1 hour after i.pl. or i.g. ethanol or acetaldehyde or their vehicles and euthanized 2 hours after ethanol or acetaldehyde. For the 4-HNE-histidine protein adduct assay, paw tissues were taken from mice euthanized before and 1, 3, 6, and 8 hours after i.g. ethanol.

For chronic alcohol administration, 1 or 2 mice were housed per cage and were initially fed the control Lieber-DeCarli diet (F1259, BioServ) ad libitum for 5 days to acclimatize them to the liquid food and tube feeding. Afterward, ethanol-fed groups were allowed free access to the ethanol Lieber-DeCarli diet containing 5\% (vol/vol) ethanol (F1258, Bio-Serv) daily for 28 days (32). Control mice were pair-fed (i.e., calorically matched to the ethanol-fed mice) with the control diet, in which equal calories of maltose-dextrin was consumed in place of ethanol (32). The feeding tubes contained 30 or $50 \mathrm{~mL}$ of ethanol or control liquid diet for 1 or 2 mice, respectively. Mice in both the experimental groups were healthy and displayed normal reflexes and alertness. A967079 and PBN (both $100 \mathrm{mg} / \mathrm{kg}$, i.p.) were administered at 
day 28 after ethanol or control liquid diet. Liver and paw tissues for ethanol and acetaldehyde assays were obtained from mice euthanized at day 28 after ethanol or control liquid diet.

\section{Acute nociceptive behavior, hind-paw mechanical allodynia, and rotarod test}

To assess acute nociceptive responses, mice were placed in a plexiglass chamber immediately after ethanol $(15 \%-80 \%$ in $0.9 \% \mathrm{NaCl}$, i.pl.), acetaldehyde (0.1-10 nmol/site in $\mathrm{NaCl}$, i.pl.), AITC (10 nmol in $0.5 \%$ DMSO, i.pl.), or their respective vehicles, and the total time (nociception time, seconds) spent in licking and lifting the injected right hind limb (21) was recorded for 5 minutes. Mechanical allodynia was evaluated by application of the von Frey filaments to the posterior hind paw of mice before (basal threshold) and after (1-24 hours) ethanol (15\%-80\% in $0.9 \% \mathrm{NaCl}$, i.pl.; $1-4 \mathrm{~mL} / \mathrm{kg}$ of $15 \%$ in $0.9 \%$ $\mathrm{NaCl}$, i.g.), acetaldehyde (1-20 nmol/site in $0.9 \% \mathrm{NaCl}$, i.pl.; 0.1-1 $\mathrm{mg} / \mathrm{kg}$ in $0.9 \% \mathrm{NaCl}$, i.p.), or their respective vehicles and in mice fed with the Lieber-DeCarli or control diet over 28 days. Mechanical threshold was determined using the up-and-down paradigm (47).

Locomotor function, coordination, and sedation of animals were tested using a rotarod apparatus (UgoBasile). Twenty-four hours before the experiments, mice were trained on the rotarod apparatus, programmed at $8 \mathrm{rpm}$, until they remained without falling for $60 \mathrm{sec}-$ onds. The day of the experiment, the latency (seconds) to the first fall and the number of falls were recorded. Cutoff time was 240 seconds.

Ethanol, acetaldehyde, $\mathrm{H}_{2} \mathrm{O}_{2}$, and 4-HNE-histidine protein adduct assays For ethanol assay, blood taken into heparin-containing syringes via direct cardiac puncture from anesthetized mice was centrifuged at $10,600 \times g$ for 5 minutes, and the plasma was collected. For ethanol and acetaldehyde assay, paw and liver tissues from euthanized mice were homogenized in PBS (0.1 M) with a tissue homogenizer (Qiagen SpA) for 30 seconds and centrifuged at 10,600 $\mathrm{g} g$ for 10 minutes, and supernatants were collected. Ethanol content was determined by a colorimetric assay (ab65343, Abcam) according to the manufacturer's protocol, and expressed as $\mathrm{nmol} / \mathrm{mL}$ (plasma) and $\mathrm{nmol} / \mathrm{mg}$ of protein (tissues). Acetaldehyde content (nmol/mg of protein) was determined by a colorimetric assay (Megazyme) according to the manufacturer's protocol.

The $\mathrm{H}_{2} \mathrm{O}_{2}$ content was determined in the paw tissue by the Amplex Red assay (Invitrogen). Briefly, tissues were rapidly placed into modified Krebs/HEPES buffer (composition in mmol/L: $99.01 \mathrm{NaCl}$, $4.69 \mathrm{KCl}, 2.50 \mathrm{CaCl}_{2}, 1.20 \mathrm{MgSO}_{4}, 1.03 \mathrm{KH}_{2} \mathrm{PO}_{4}, 25.0 \mathrm{NaHCO}_{3}, 20.0$ Na-HEPES, and 5.6 glucose, $\mathrm{pH} 7.4)$, minced, and incubated with Amplex Red $(100 \mu \mathrm{M})$ and HRP $(1 \mathrm{U} / \mathrm{mL})\left(1\right.$ hour, $\left.37^{\circ} \mathrm{C}\right)$ in modified Krebs/HEPES buffer protected from light (48). Fluorescence excitation and emission were at 540 and $590 \mathrm{~nm}$, respectively. $\mathrm{H}_{2} \mathrm{O}_{2}$ production was calculated using $\mathrm{H}_{2} \mathrm{O}_{2}$ standard and expressed as $\mu \mathrm{mol} / \mathrm{L} /$ mg of dry tissue.

4-HNE-histidine (4-HNE-His) protein adduct content was quantified in paw tissues by a colorimetric assay (OxiSelect HNE-His Adduct ELISA Kit, Cell Biolabs Inc.) according to the manufacturer's protocol. Paw tissues were homogenized in PBS (0.1 M), supplemented with proteases (serine and cysteine metalloproteinases and calpains) inhibitors ( 1 tablet/100 $\mathrm{mL}$ ) for 30 seconds, and centrifuged (10,600 $\mathrm{x} g, 10$ minutes, $\left.4^{\circ} \mathrm{C}\right)$. Supernatants were assayed for the measurement of total protein content by a Bradford assay (Bio-Rad) and 4-HNE-His content (expressed as $\mu \mathrm{g} / \mathrm{mg}$ of protein).

\section{Cell cultures}

Human embryonic kidney 293 (HEK293) cells stably transfected with the cDNA for human TRPA1 (hTRPA1-HEK293) were cultured as previously described (49). Human Schwann cells (HSCs) (1700, CliniScience) were cultured in Schwann cell medium (1701, CliniScience) according to the manufacturer's protocol. Human alveolar type II epithelium-like adherent cell line (A549) (CCL-185, American Type Culture Collection) was cultured in RPMI with $10 \%$ FBS, 2 mM glutamine, $100 \mathrm{U}$ penicillin, $100 \mu \mathrm{g} / \mathrm{mL}$ streptomycin, and $1 \mathrm{mM}$ HEPES. All cells were used when received without further authentication.

Schwann cells were isolated from sciatic nerves of C57BL/6J mice. The epineurium was removed, and nerve explants were divided into 1-mm segments and dissociated enzymatically using collagenase $(0.05 \%)$ and hyaluronidase $(0.1 \%)$ in HBSS $\left(2\right.$ hours, $\left.37^{\circ} \mathrm{C}\right)$. Cells were collected by centrifugation ( $68 \mathrm{x} \mathrm{g}, 10$ minutes, room temperature), and the pellet was resuspended and cultured in DMEM containing $10 \%$ FCS, $2 \mathrm{mM}$ L-glutamine, $100 \mathrm{U} / \mathrm{mL}$ penicillin, and $100 \mathrm{mg} / \mathrm{mL}$ streptomycin. Three days later, cytosine arabinoside $(10 \mathrm{mM})$ was added to remove fibroblasts. All cells were cultured in an atmosphere of $95 \%$ air and $5 \% \mathrm{CO}_{2}$ at $37^{\circ} \mathrm{C}$.

\section{Assays in cultured cells}

Calcium imaging. HSCs were plated on glass coverslips coated with poly-L-lysine $(8.3 \mu \mathrm{M})$, and $\left[\mathrm{Ca}^{2+}\right]_{\mathrm{i}}$ response was measured as previously reported (22). HSCs were exposed to AITC $(100 \mu \mathrm{M}), \mathrm{H}_{2} \mathrm{O}_{2}(10 \mathrm{mM})$, and acetaldehyde $(10 \mathrm{mM})$ or their vehicles $(0.1 \% \mathrm{DMSO}$ or $0.9 \% \mathrm{NaCl})$ in the presence or absence of A967079 $(50 \mu \mathrm{M})$ or its vehicle $(0.3 \%$ DMSO). In other experiments, HSCs were exposed to ethanol (10 mM) or its vehicle. In this case the delayed $\left[\mathrm{Ca}^{2+}\right]_{\mathrm{i}}$ response was monitored for approximately 150 minutes in the presence or absence of A967079 $(50 \mu \mathrm{M})$ or $4-\mathrm{Mp}(100 \mu \mathrm{M})$ or their vehicle (0.3\% DMSO). Results were expressed as percent increase in ratio ${ }_{340 / 380}$ over baseline normalized to the maximum effect induced by ionomycin $(5 \mu \mathrm{M})$ added at the end of each experiment.

Acetaldehyde assay. Mouse Schwann cells and HSCs were plated in 48 -well plates in $5 \% \mathrm{CO}_{2}$ and $95 \% \mathrm{O}_{2}$ at $37^{\circ} \mathrm{C}$ until $90 \%$ of confluence. The cultured medium was replaced with PBS supplemented with ethanol $(1-100 \mathrm{mM})$, ethanol $(10 \mathrm{mM})$ plus $4-\mathrm{Mp}(100 \mu \mathrm{M})$, or vehicle $(0.9 \% \mathrm{NaCl})$ and maintained in $5 \% \mathrm{CO}_{2}$ and $95 \% \mathrm{O}_{2}$ at $37^{\circ} \mathrm{C}$ for 3 hours. Then, supernatants were collected, and acetaldehyde content was assayed by a colorimetric assay (Megazyme) according to the manufacturer's protocol. Acetaldehyde content was expressed as micromoles per liter. The cytotoxicity in response to different stimuli was tested by the MTT assay viability test.

$\mathrm{H}_{2} \mathrm{O}_{2}$ assay. $\mathrm{H}_{2} \mathrm{O}_{2}$ was determined by the Amplex Red assay. HSCs were plated in 96-well clear-bottom black plates $\left(5 \times 10^{5}\right.$ cells per well) and maintained in $5 \% \mathrm{CO}_{2} / 95 \% \mathrm{O}_{2}\left(24\right.$ hours, $\left.37^{\circ} \mathrm{C}\right)$. The cultured medium was replaced with Krebs/HEPES supplemented with A967079 $(30 \mu \mathrm{M})$ or vehicle $(0.3 \%$ DMSO) for 10 minutes at room temperature. HSCs were stimulated with AITC $(100 \mu \mathrm{M}), \mathrm{H}_{2} \mathrm{O}_{2}(200$ $\mathrm{nM})$, and acetaldehyde $(10 \mathrm{mM})$ or their vehicle (0.01\% DMSO, Krebs/ HEPES) supplemented with Amplex Red $(50 \mu \mathrm{M})$ and HRP $(1 \mathrm{U} / \mathrm{mL}$, 30 minutes, room temperature, protected from light). Some experiments were performed in $\mathrm{Ca}^{2+}$-free Krebs/HEPES buffer containing EDTA $(1 \mathrm{mM})$. Signal was detected 60 minutes after exposure to stimuli. $\mathrm{H}_{2} \mathrm{O}_{2}$ release was calculated using $\mathrm{H}_{2} \mathrm{O}_{2}$ standards and expressed as nanomoles per liter. 


\section{Immunofluorescence}

Anesthetized mice were transcardially perfused with PBS and $4 \%$ paraformaldehyde. Paw tissues were removed, postfixed for 24 hours, and paraffin-embedded. Human and mouse FFPE sections $(5 \mu \mathrm{m})$ were incubated with primary antibodies (all from Abcam): TRPA1 (ab58844, rabbit polyclonal, 1:400), S100 (ab14849, mouse monoclonal [4B3], 1:300), protein gene product 9.5 (PGP9.5) (ab8189, mouse monoclonal [13C4/I3C4], 1:600), NOX1 (ab131088, rabbit polyclonal, 1:250), 4-HNE (HNEJ-2, ab48506, mouse monoclonal, 1:40), and ADH (ab108203, rabbit monoclonal [EPR4439], 1:200), diluted in fresh blocking solution (PBS, pH 7.4, 2.5\% normal goat serum, [NGS]). To confirm specificity, TRPA1, NOX1, and ADH primary antibodies were preadsorbed $\left(1: 1\right.$, overnight, $4^{\circ} \mathrm{C}$, before adding to tissue sections) with their respective antigen peptides: TRPA1 synthetic peptide (sequence: CEKQHELIKLIIQKME; Twin Helix Srl.), $\mathrm{ADH}$ recombinant protein (NBP1-99053, Novus Biologicals), and NOX1 recombinant protein (PCPKAB7187921P, Promocell). Sections were then incubated with the fluorescent polyclonal secondary antibodies Alexa Fluor 488 and 594 (1:600; Invitrogen), and coverslipped using mounting medium with DAPI (Abcam). Image of the product of the differences from the mean (PDM), i.e. for each pixel (red intensity - mean red intensity) $\times$ (green intensity - mean green intensity), was used for qualitative analysis of colocalization. The PDM image is pseudo-colored, generated by pixel that is equal to the PDM value at that location (ImageJ, NIH). The orange color indicates colocalized pixels and the blue color means segregation.

HSCs were grown on glass coverslips coated with poly-L-lysine $(8.3 \mu \mathrm{M})$ and cultured for 2-3 days before staining. Cells were fixed in ice-cold methanol/acetone ( 5 minutes at $-20^{\circ} \mathrm{C}$ ), washed with PBS, and blocked with NGS (10\%) (1 hour, room temperature). The cells were incubated with primary antibodies: TRPA1 (ab58844, rabbit polyclonal, 1:400), S100 (ab14849, mouse monoclonal [4B3], 1:300), NOX1 (ab131088, rabbit polyclonal, 1:250), and ADH (ab108203, rabbit monoclonal [EPR4439], 1:200), diluted in fresh blocking solution (PBS, pH 7.4, 2.5\% NGS). Cells were finally incubated with fluorescent polyclonal secondary antibodies (1:600; Alexa Fluor 488 and 594, Invitrogen) (2 hours, room temperature) and mounted using mounting medium with DAPI (Abcam). Fluorescence images were obtained using a BX51 microscope (Olympus).

\section{Immunohistochemistry and intraepidermal nerve fiber analysis}

Anesthetized mice were transcardially perfused with PBS and $4 \%$ paraformaldehyde. Mouse paws were placed overnight at $4^{\circ} \mathrm{C}$ in $10 \%$ formalin, transferred to $30 \%$ sucrose overnight, and frozen and cryosectioned at $30 \mu \mathrm{m}$ transversal to long paw axis. Free-floating sections were incubated in PBS containing 0.1\% Triton X-100 (TBS) and 2.5\% NGS 1 hour at room temperature, then in the primary antibody panaxonal marker PGP9.5 (1:600; ab108986, rabbit monoclonal [EPR4118], Abcam) overnight at room temperature. Afterward, sections were rinsed in TBS, placed in secondary biotinylated goat anti-rabbit IgG antibody (Vector Laboratories) (1:300, 2 hours at room temperature), and then placed in avidin-biotin complex solution (Vectastain Elite ABC HRP, Vector Laboratories) for 30 minutes at room temperature, followed by rinsing in TBS. The sections were then transferred into ImmPACT DAB (Vector Laboratories) for 4-6 minutes for the chromogen development reaction and rinsed in distilled water before mounting. Intraepidermal nerve fibers (IENFs) were counted under a microscope at $\times 40$ magnification. Single PGP9.5-positive fibers crossing the epidermis-dermis boundary (basal membrane) were counted. Secondary branching was excluded from quantification, according to the European Federation of Neurological Societies guidelines (50).

\section{Real-time PCR}

RNA was extracted from paw tissue and liver obtained from C57BL/6J mice or from HSCs. Total RNA was extracted using the RNeasy Mini kit (Qiagen SpA), according to the manufacturer's protocol. RNA concentration and purity were assessed spectrophotometrically. Reverse transcription was performed with the Qiagen QuantiTect Reverse Transcription Kit (Qiagen SpA) following the manufacturer's protocol. For mRNA relative quantification, real-time PCR was performed on Rotor Gene Q (Qiagen SpA). The sets of probes for human cells were: ACTB ( $\beta$-actin): primer 1, CCTTGCACATGCCGGAG; primer 2, ACAGAGCCTCGCCTTTG; probe, /56-FAM/TCATCCATG/ZEN/GTGAGCTGGCGG/3IABkFQ/ (NCBI reference sequence [ref. seq.] NM_001101); TRPA1: primer 1, GAAACCAAAGTGGCAGCTTC; primer 2, GACATTGCTGAGGTCCAGAA; probe, /56-FAM/TGAAGTTCC/ZEN/ACCTGCATAGCTATCCTCT/3IABkFQ/ (NCBI ref. seq. NM_007332); NOX1: primer 1, AAACATTCAGCCCTAACCAAAC; primer 2, GAATCTTCCCTGTTGCCTAGA; probe, /56-FAM/ACCACCCAG/ZEN/TTTCCCATTGTCAAGA/3IABkFQ/ (NCBI ref. seq. NM013955); ADH1A: primer 1, GTTTCTTTAACTCCCATAGCACAG; primer 2, CACAAGGACTCACCAGTCTC; probe, /56-FAM/AGACAGAAT/ZEN/CAACATGAGCACAGCAGG/3IABkFQ/ (NCBI ref. seq. NM_000667); ADH1B: primer 1, GTGCTCATGTCGTTTCTGTCT; primer 2, TCAAGCAGAGAAGAAATCCACAA; probe, /56-FAM/TGCCCACCA/ZEN/ GCAGACTGTGA/3IABkFQ/ (NCBI ref. seq. NM_000668); ADH1C: primer 1, ATCTTAATGCGAACTTCATGAGC; primer 2, CAGAATCAATATGAGCACAGCAG; probe, /56-FAM/CCATTGAGG/ZEN/ AGGTAGAGGTTGCACC/3IABkFQ/ (NCBI ref. seq. NM_000669); ADH4: primer 1, AGAAAGACCCACACCTCCTA; primer 2, GAGTTTGTCTGCTTGGATGTG; probe, /56-FAM/CCCTGGTTC/ ZEN/GACTTGTGCTGTCT/3IABkFQ/ (NCBI ref. seq. NM_000670); ADH5: primer 1, TGCCACCTCTATCTCCTCTATG; primer 2, CCGACCAGAATCCGTGAAC; probe, /56-FAM/AGCCTTGCA/ZEN/CTTGATAACCTCGTTCG/3IABkFQ/ (NCBI ref. seq. NM_000671); ADH6: primer 1, TGTGGCAGAAAGAGTGTGAT; primer 2, CCTCTTGTATCCCACCATCTTG; probe, /56-FAM/TCACCTGGT/ZEN/TTCACTGTGCTTACTCC/3IABkFQ/ (NCBI ref. seq. NM_000672).

The sets of probes for mouse tissue were: ACTB: primer 1, GACTCATCGTACTCCTGCTTG; primer 2, GATTACTGCTCTGGCTCCTAG; probe, /56-FAM/CTGGCCTCA/ZEN/CTGTCCACCTTCC/3IABkFQ/ (NCBI ref. seq. NM_001101); ADH1: primer 1, AAGACTACAGCAAACCCATCC; primer 2, GACGACGCTTACACCACAT; probe, /56-FAM/CCTTGACAC/ZEN/CATGACTTCTGCCCT/31ABkFQ/ (NCBI ref. seq. NM_007409); ADH5: primer 1, TCATCCCACTCTACATCCCA; primer 2, GGTAAATCTGCTAGTCCCATCT; probe, /56-FAM/CCCTTCCCC/ZEN/TGAGTGACCCTTATTTTC/31ABkFQ/ (NCBI ref. seq. NM_007410); ADH7: primer 1, TGAAGTTATTGGGCGTCTTGA; primer 2, GTCATAGGTGAGCATCTTGGC; probe, /56-FAM/CAGTGTGGT/ZEN/GGTTGGTGCTCCT/31ABkFQ/ (NCBI ref. seq. NM_009626).

The chosen reference gene was ACTB. The QuantiTect Probe PCR Kit (Qiagen SpA) was used for amplification, and the cycling 
conditions were as follows: samples were heated to $95^{\circ} \mathrm{C}$ for $10 \mathrm{~min}$ utes followed by 40 cycles of $95^{\circ} \mathrm{C}$ for 15 seconds and $65^{\circ} \mathrm{C}$ for $20 \mathrm{sec}-$ onds. PCR reaction was carried out in triplicate. Relative expression of mRNA was calculated using the $2^{-\Delta(\triangle C T)}$ comparative method, with each gene normalized against the ACTB gene for the same sample.

\section{Statistics}

Statistical analysis was performed by the unpaired 2-tailed Student's $t$ test for comparisons between 2 groups. Group means were compared with a 1-way ANOVA, followed, as needed, by pairwise comparison of multiple groups that used Bonferroni's correction to maintain the experiment-wise error rate at 5\%. For behavioral experiments with repeated measures, a 2-way mixed model was used to compare the control and treated groups of mice at each time point tested, using Bonferroni's correction for multiple time points. Statistical analyses were performed on raw data using Prism 5 software (GraphPad Software Inc.). $P$ less than 0.05 was considered statistically significant.

\section{Study approval}

The use of FFPE sections of human skin samples was approved by the Local Ethics Committee of the Careggi University Hospital (11989_ bio/2018), according to the Helsinki Declaration, and informed consent was obtained. All applicable international, national, and/ or institutional guidelines for the care and use of animals were followed. In vivo experiments were in accordance with European Union
(EU) guidelines and Italian legislation (DLgs 26/2014, EU Directive application 2010/63/EU) for animal care procedures, and under University of Florence research permit 194/2015-PR. Animal studies were reported in compliance with the ARRIVE guidelines (51).

\section{Author contributions}

FDL, RP, PG, and RN designed research studies. FDL, SLP, LL, FP, DSMDA, and RN conducted experiments. FDL, SLP, LL, FP, DSMDA, and RN acquired data. FDL, MNJ, and RN analyzed data. AI provided human tissue samples. FDL, MNJ, RP, NWB, PG, and RN wrote the manuscript.

\section{Acknowledgments}

We thank A.H. Morice (University of Hull, Hull, UK) for hTRPA1-HEK293 cells and D. Preti (University of Ferrara, Ferrara, Italy) for providing A967079. We also thank Mary K. Lokken for her expert English revision. This study was funded by Ministery of Education, University and Research, MIUR (Rome, Italy) grant PRIN 201532AHAE_003 (to PG), and by grants from the NIH (NS102722, DE026806, DK118971) and the Department of Defense (W81XWH1810431) (to NWB).

Address correspondence to: Pierangelo Geppetti, Department of Health Sciences, University of Florence, Viale Pieraccini 6, 50139 Florence, Italy. Phone: 39.349.271.0476; Email: geppetti@unifi.it.
1. Rehm J, Mathers C, Popova S, Thavorncharoensap M, Teerawattananon Y, Patra J. Global burden of disease and injury and economic cost attributable to alcohol use and alcohol-use disorders. Lancet. 2009;373(9682):2223-2233.

2. WHO. Global Status Report on Alcohol Health 2018. Geneva, Switzerland: WHO Press; 2018.

3. Chopra K, Tiwari V. Alcoholic neuropathy: possible mechanisms and future treatment possibilities. Br J Clin Pharmacol. 2012;73(3):348-362.

4. Singleton CK, Martin PR. Molecular mechanisms of thiamine utilization. Curr Mol Med. 2001;1(2):197-207.

5. Mellion M, Gilchrist JM, de la Monte S. Alcoholrelated peripheral neuropathy: nutritional, toxic, or both? Muscle Nerve. 2011;43(3):309-316.

6. Koike H, et al. Postgastrectomy polyneuropathy with thiamine deficiency. J Neurol Neurosurg Psychiatry. 2001;71(3):357-362.

7. Koike H, et al. Alcoholic neuropathy is clinicopathologically distinct from thiamine-deficiency neuropathy. Ann Neurol. 2003;54(1):19-29.

8. Cederbaum AI. Alcohol metabolism. Clin Liver Dis. 2012;16(4):667-685.

9. Eriksson CJ. The role of acetaldehyde in the actions of alcohol (update 2000). Alcohol Clin Exp Res. 2001;25(5 suppl ISBRA):15S-32S.

10. Andrici J, Hu SX, Eslick GD. Facial flushing response to alcohol and the risk of esophageal squamous cell carcinoma: a comprehensive systematic review and meta-analysis. Cancer Epidemiol. 2016;40:31-38.

11. Bagnardi V, et al. Light alcohol drinking and cancer: a meta-analysis. Ann Oncol. 2013;24(2):301-308.

12. Boffetta P, Hashibe M. Alcohol and cancer. Lancet Oncol. 2006;7(2):149-156.
13. Xiao Q, Weiner H, Crabb DW. The mutation in the mitochondrial aldehyde dehydrogenase (ALDH2) gene responsible for alcohol-induced flushing increases turnover of the enzyme tetramers in a dominant fashion. J Clin Invest. 1996;98(9):2027-2032.

14. Wall TL, et al. Alcohol metabolism in Asian-American men with genetic polymorphisms of aldehyde dehydrogenase. Ann Intern Med.1997;127(5):376-379.

15. Chen CH, Ferreira JC, Gross ER, MochlyRosen D. Targeting aldehyde dehydrogenase 2: new therapeutic opportunities. Physiol Rev. 2014;94(1):1-34.

16. Trevisani M, et al. Ethanol elicits and potentiates nociceptor responses via the vanilloid receptor-1. Nat Neurosci. 2002;5(6):546-551.

17. Andersson DA, Gentry C, Moss S, Bevan S. Transient receptor potential A1 is a sensory receptor for multiple products of oxidative stress. J Neurosci. 2008;28(10):2485-2494.

18. Takahashi N, et al. TRPA1 underlies a sensing mechanism for O2. Nat Chem Biol. 2011;7(10):701-711.

19. Nassini R, Materazzi S, Benemei S, Geppetti P. The TRPA1 channel in inflammatory and neuropathic pain and migraine. Rev Physiol Biochem Pharmacol. 2014;167:1-43.

20. Bang S, Kim KY, Yoo S, Kim YG, Hwang SW. Transient receptor potential A1 mediates acetaldehyde-evoked pain sensation. Eur J Neurosci. 2007;26(9):2516-2523.

21. Trevisani M, et al. 4-Hydroxynonenal, an endogenous aldehyde, causes pain and neurogenic inflammation through activation of the irritant receptor TRPA1. Proc Natl Acad Sci US A.
2007;104(33):13519-13524

22. De Logu F, et al. Schwann cell TRPA1 mediates neuroinflammation that sustains macrophage-dependent neuropathic pain in mice. Nat Commun. 2017;8(1):1887.

23. Galter D, Carmine A, Buervenich S, Duester G, Olson L. Distribution of class I, III and IV alcohol dehydrogenase mRNAs in the adult rat, mouse and human brain. Eur J Biochem. 2003;270(6):1316-1326.

24. Zgombić-Knight M, Ang HL, Foglio MH, Duester G. Cloning of the mouse class IV alcohol dehydrogenase (retinol dehydrogenase) cDNA and tissue-specific expression patterns of the murine $\mathrm{ADH}$ gene family. J Biol Chem. 1995;270(18):10868-10877.

25. Seitz HK, Meier P. The role of acetaldehyde in upper digestive tract cancer in alcoholics. Transl Res. 2007;149(6):293-297.

26. Zakhari S. Overview: how is alcohol metabolized by the body? Alcohol Res Health. 2006;29(4):245-254.

27. Szuster-Ciesielska A, Plewka K, Daniluk J, Kandefer-Szerszeń M. Zinc supplementation attenuates ethanol- and acetaldehyde-induced liver stellate cell activation by inhibiting reactive oxygen species (ROS) production and by influencing intracellular signaling. Biochem Pharmacol.2009;78(3):301-314.

28. Hoyt LR, et al. Mitochondrial ROS induced by chronic ethanol exposure promote hyperactivation of the NLRP3 inflammasome. Redox Biol. 2017;12:883-896.

29. Vrsalovic M, Vrsalovic MM, Presecki AV, Lukac J. Modulating role of alcohol and acetaldehyde on neutrophil and monocyte functions in vitro. J Cardiovasc Pharmacol. 2007;50(4):462-465. 
30. Aldini G, Carini M, Yeum KJ, Vistoli G. Novel molecular approaches for improving enzymatic and nonenzymatic detoxification of 4-hydroxynonenal: toward the discovery of a novel class of bioactive compounds. Free Radic Biol Med. 2014;69:145-156.

31. Marone IM, et al. TRPA1/NOX in the soma of trigeminal ganglion neurons mediates migraine-related pain of glyceryl trinitrate in mice. Brain. 2018;141(8):2312-2328.

32. Bertola A, Mathews S, Ki SH, Wang H, Gao B. Mouse model of chronic and binge ethanol feeding (the NIAAA model). Nat Protoc. 2013;8(3):627-637.

33. Dalle-Donne I, Aldini G, Carini M, Colombo R, Rossi R, Milzani A. Protein carbonylation, cellular dysfunction, and disease progression. JCell MolMed.2006;10(2):389-406.

34. Gianni D, et al. A novel and specific NADPH oxidase-1 (Nox1) small-molecule inhibitor blocks the formation of functional invadopodia in human colon cancer cells. ACS Chem Biol. 2010;5(10):981-993.

35. Boyette-Davis J, Dougherty PM. Protection against oxaliplatin-induced mechanical hyperalgesia and intraepidermal nerve fiber loss by minocycline. Exp Neurol. 2011;229(2):353-357.

36. Bianchi R, et al. Erythropoietin both protects from and reverses experimental diabetic neuropathy. Proc Natl Acad Sci U S A. 2004;101(3):823-828.

37. Obrosova IG, et al. PARP inhibition or gene deficiency counteracts intraepidermal nerve fiber loss and neuropathic pain in advanced diabetic neuropathy. Free Radic Biol Med. 2008;44(6):972-981.

38. Koivisto A, et al. Inhibiting TRPA1 ion channel reduces loss of cutaneous nerve fiber function in diabetic animals: sustained activation of the TRPA1 channel contributes to the pathogenesis of peripheral diabetic neuropathy. Pharmacol Res. 2012;65(1):149-158.

39. Mellion ML, Silbermann E, Gilchrist JM, Machan JT, Leggio L, de la Monte S. Small-fiber degeneration in alcohol-related peripheral neuropathy. Alcohol Clin Exp Res. 2014;38(7):1965-1972.

40. Zambelis T, Karandreas N, Tzavellas E, Kokotis P, Liappas J. Large and small fiber neuropathy in chronic alcohol-dependent subjects. J Peripher Nerv Syst. 2005;10(4):375-381.

41. Hamilton NB, Kolodziejczyk K, Kougioumtzidou E, Attwell D. Proton-gated Ca(2+)-permeable TRP channels damage myelin in conditions mimicking ischaemia. Nature. 2016;529(7587):523-527.

42. Kwan KY, et al. TRPA1 contributes to cold, mechanical, and chemical nociception but is not essential for hair-cell transduction. Neuron. 2006;50(2):277-289.

43. Liedtke W, Friedman JM. Abnormal osmotic regulation in trpv4-/- mice. Proc Natl Acad Sci US A. 2003;100(23):13698-13703.

44. Guan Z, et al. Injured sensory neuron-derived CSF1 induces microglial proliferation and DAP12-dependent pain. Nat Neurosci. 2016;19(1):94-101.
45. Zappia KJ, O’Hara CL, Moehring F, Kwan KY, Stucky CL. Sensory neuron-specific deletion of TRPA1 results in mechanical cutaneous sensory deficits. eNeuro. 2017;4(1):ENEURO.0069-16.2017.

46. Faul F, Erdfelder E, Buchner A, Lang AG. Statistical power analyses using $G^{*}$ Power 3.1: tests for correlation and regression analyses. Behav Res Methods. 2009;41(4):1149-1160.

47. Dixon WJ. Efficient analysis of experimental observations. Annu Rev Pharmacol Toxicol. 1980;20:441-462.

48. Landmesser U, et al. Oxidation of tetrahydrobiopterin leads to uncoupling of endothelial cell nitric oxide synthase in hypertension. J Clin Invest. 2003;111(8):1201-1209.

49. Sadofsky LR, et al. Unique responses are observed in transient receptor potential Ankyrin 1 and Vanilloid 1 (TRPA1 and TRPV1) co-expressing cells. Cells. 2014;3(2):616-626.

50. Lauria G, et al. European Federation of Neurological Societies/Peripheral Nerve Society Guideline on the use of skin biopsy in the diagnosis of small fiber neuropathy. Report of a joint task force of the European Federation of Neurological Societies and the Peripheral Nerve Society. Eur J Neurol. 2010;17(7):903-e49.

51. Kilkenny C, Browne WJ, Cuthill IC, Emerson M, Altman DG. Improving bioscience research reporting: the ARRIVE guidelines for reporting animal research. J Pharmacol Pharmacother. 2010;1(2):94-99. 